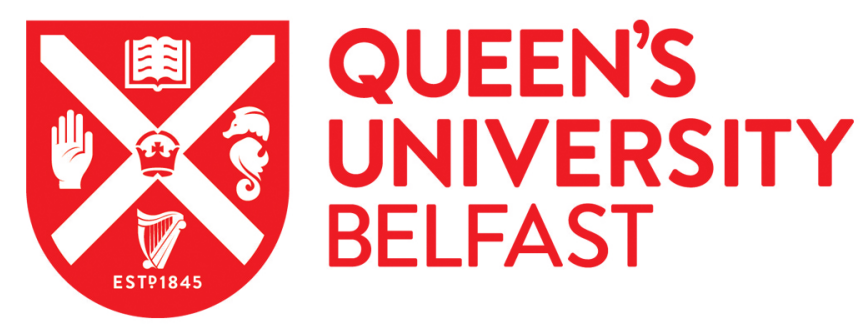

\title{
Tensor-Based Algebraic Channel Estimation for Hybrid IRS-Assisted MIMO-OFDM
}

Lin, Y., Jin, S., Matthaiou, M., \& You, X. (2021). Tensor-Based Algebraic Channel Estimation for Hybrid IRSAssisted MIMO-OFDM. IEEE Transactions on Wireless Communications. https://doi.org/10.1109/TWC.2021.3053580

Published in:

IEEE Transactions on Wireless Communications

Document Version:

Peer reviewed version

Queen's University Belfast - Research Portal:

Link to publication record in Queen's University Belfast Research Portal

Publisher rights

(c) 2021 IEEE.

This work is made available online in accordance with the publisher's policies. Please refer to any applicable terms of use of the publisher.

\section{General rights}

Copyright for the publications made accessible via the Queen's University Belfast Research Portal is retained by the author(s) and / or other copyright owners and it is a condition of accessing these publications that users recognise and abide by the legal requirements associated with these rights.

Take down policy

The Research Portal is Queen's institutional repository that provides access to Queen's research output. Every effort has been made to ensure that content in the Research Portal does not infringe any person's rights, or applicable UK laws. If you discover content in the Research Portal that you believe breaches copyright or violates any law, please contact openaccess@qub.ac.uk. 


\title{
Tensor-Based Algebraic Channel Estimation for Hybrid IRS-Assisted MIMO-OFDM
}

\author{
Yuxing Lin, Student Member, IEEE, Shi Jin, Senior Member, IEEE, Michail Matthaiou, Senior Member, IEEE, \\ and Xiaohu You, Fellow, IEEE
}

\begin{abstract}
We consider the channel estimation problem in multiple-input multiple-output orthogonal frequency division multiplexing (MIMO-OFDM) systems assisted by intelligent reconfigurable surfaces (IRSs). To avoid the inherent estimation ambiguities of the two-hop channels from mobile stations (MS) to the base station (BS), we adopt a hybrid IRS architecture composed of passive reflectors and active sensors, and establish two independent subproblems of estimating the MS-to-IRS and BS-to-IRS channels. By leveraging the sparse characteristics of high-frequency propagation, we model the training signals as multi-dimensional canonical polyadic decomposition (CPD) tensors with missing fibers or slices. We develop algebraic algorithms to solve the tensor completion problems and recover channel multipath parameters, i.e., angles of arrival, time delays and path gains. Our methods require neither random initialization nor iterative operations, and for these reasons they can perform robustly with a low computational complexity. Moreover, we investigate the uniqueness condition of CPD tensor completion, which can be utilized to inform both the physical design of hybrid IRSs and the time-frequency resource allocation of training strategies. Simulation results indicate that the proposed schemes outperform the traditional counterparts in terms of accuracy, robustness and complexity, especially for the case of low-complexity IRSs with limited number of active sensing elements.
\end{abstract}

Index Terms-Channel estimation, CPD tensor completion, intelligent reconfigurable surfaces, MIMO-OFDM.

\section{INTRODUCTION}

In the upcoming phases of the fifth generation $(5 \mathrm{G})$ wireless communications, millimeter wave (mmWave) (30-300 GHz) transmission technologies have been widely identified as a promising solution against the increasing data traffic and frequency spectrum shortage [1]. Moreover, extending the spectrum to higher frequency bands, i.e., terahertz $(0.1-10$ $\mathrm{THz}$ ), appears to be a significant evolution direction of the future beyond-5G (B5G) and sixth generation (6G) development [2]. High-frequency radio systems avail of strong

Manuscript received May 22, 2020; revised September 17, 2020, and November 27, 2020; accepted January 7, 2021. The work of S. Jin was supported in part by the National Science Foundation of China (NSFC) for Distinguished Young Scholars under Grant 61625106 and the NSFC under Grant 61941104 . The work of M. Matthaiou was supported by Engineering and Physical Sciences Research Council (EPSRC) of U.K. under Grant EP/P000673/1. The associated editor coordinating the review of this paper and approving it for publication was Dr. Giovanni Geraci. (Corresponding authors: Xiaohu You; Shi Jin.)

Y. Lin, S. Jin, and X. You are with the National Mobile Communications Research Laboratory, Southeast University, Nanjing, P.R. China, (e-mail: yxlin@ @eu.edu.cn; jinshi@seu.edu.cn; xhyu@ seu.edu.cn).

M. Matthaiou is with the Institute of Electronics, Communications and Information Technology (ECIT), Queen's University Belfast, Belfast, BT3 9DT, U.K., (e-mail: m.matthaiou@qub.ac.uk). beam directivity, high spatial resolution and robust antiinterference characteristics, while their small wavelength enables the miniaturized implementation of massive multipleinput multiple-output (MIMO) antenna arrays to compensate for the exacerbated free-space losses [3]. However, the quasioptical mmWave and terahertz waves inherently suffer from limited coverage caused by the higher probability of lineof-sight $(\mathrm{LoS})$ propagation obstruction. In order to maintain the communication link and broaden the signal coverage area, wireless systems need to exploit the sparse scattering nonLOS (NLoS) paths of high-frequency channels. One feasible choice is to integrate the massive MIMO transceiver within an intelligent reconfigurable surface (IRS), which can help establish controllable supplementary links [4].

An IRS has been considered as a revolutionizing technology for assisting the implementation of broadband connectivity in future 6G wireless systems [5]. It can be utilized to improve the propagation conditions by introducing additional scattering with controllable characteristics to achieve desirable beamforming gains and suppress co-channel interference [6]. Ideally, an IRS can pave the way to realizing a smart and reconfigurable wireless propagation environment, which brings extra degrees of freedom to the transceiver design, as well as, the network optimization [7]. Typically, an IRS is made of a programmable metasurface, which comprises of a massive number of unit cells that can independently interact with the incident signals [8]. The reflection amplitudes or phase shifts of the IRS elements can be predefined or adjusted by a smart digital controller to realize real-time manipulation on the electromagnetic responses of the reflected waves [9]. Based on the considerable capability of optimizing the transmission links, the IRS technology can be integrated into numerous wireless applications, e.g., physical layer security, simultaneous wireless information and power transfer, cognitive radio and nonorthogonal multiple access [10]-[13]. Nevertheless, in order to fully reap the potential of IRS in communication services, it is necessary for the IRS controller to obtain accurate channel state information (CSI) of the channel matrices or multipath parameters. This critical exercise relies on feasible channel estimation strategies.

There have been numerous works researching the channel estimation problem of IRS-assisted systems. A binary reflection controlled least squares scheme was proposed in [14], enabling the IRS switch function to element-wisely estimate the cascaded channel. A joint bilinear factorization and matrix completion scheme was proposed in [15], combining the approximate message passing technology and Riemannian gradi- 
ent algorithm. A two-stage sounding method with atomic norm minimization optimization was designed in [16] to recover the cascaded channel parameters. Compressed sensing (CS) tools were adopted by [17] to exploit the row-column-block sparsity of the cascaded multipath channel. Hierarchical beamforming codebook as well as cooperative search strategies were proposed in [18]. A deep learning framework containing a twin convolutional neural network was applied in [19] to jointly estimate the direct and cascaded channels. Tensor signal processing methods were also leveraged in [20], [21] to solve the bilinear channel recovery problem. An anchor-assisted method was proposed in [22] to efficiently reduce the channel training overhead with one-antenna anchors. Most works aims to directly estimate the cascaded two-hop channel, which inherently causes scaling ambiguities between the MS-to-IRS and IRS-to-BS channels. Moreover, they commonly consider flat-fading channel models, which, unfortunately, cannot be directly applied to the dynamic time-varying channel links.

In this paper, we precisely address the channel estimation problem of an IRS-assisted MIMO orthogonal frequency division multiplexing (OFDM) system operating at mmWave frequencies. We propose a hybrid IRS structure composed of passive reflectors and active sensors, which can simultaneously reflect the signal waves and sense the channels [23], [24]. This architecture can be equivalently regarded as a hybrid layout consisting of one or multiple IRS planes and antenna anchors/arrays connected to radio frequency chains (RFC), where the reflecting/sensing modules jointly formulate a regular planar topology. The main contributions of this paper are summarized as follows:

- For the hybrid IRS structure, we factorize the cascaded channel estimation problem into two subproblems of recovering the MS-to-IRS and BS-to-IRS channels. The downlink/uplink training can be simultaneously performed through non-overlapping frequency bands.

- For the narrowband channel estimation with single training subcarrier, we leverage the sparsity of high-frequency propagation to formulate the received signals as a thirdorder canonical polyadic decomposition (CPD) tensor [25]-[27]. We transform the channel estimation task to complete a partially-observed tensor [28]-[30], and develop an algebraic algorithm to jointly recover the channel matrix and multipath parameters. ${ }^{1}$

- For the wideband channel estimation with multiple training subcarriers, we formulate the received signals as a fourth-order incomplete CPD tensor. By leveraging the structural information of training signal in the frequency domain, we propose two different algorithms to solve the tensor completion problem.

- We analyze the uniqueness conditions of the third/fourthorder CPD tensor completions, and propose practical suggestions on the hybrid IRS configuration and channel training design.

\footnotetext{
${ }^{1}$ The algebraic tensor method does not restrict the IRS array structure and the pilot symbol arrangement, requiring neither statistical derivation nor optimization procedure. Also, most involved operations are supported by ready-made toolboxes/libraries.
}

Simulation results indicate that the proposed schemes outperform the traditional methods (e.g., those proposed in [23], [28]-[30]) in terms of accuracy, robustness and complexity, especially for the case where the number of active IRS elements are extremely limited.

The rest of the paper is organized as follows. Section II presents the IRS-assisted MIMO-OFDM system model and the high-frequency channel model. Section III develops the narrowband channel estimation algorithm with single training subcarrier. Section IV proposes the wideband channel estimation algorithms with multiple training subcarriers. Section V presents the numerical results of the estimation algorithms. Section VI draws the most important conclusions.

Notations: a, $\mathbf{A}$ and $\mathcal{A}$ denote a vector, a matrix and a tensor, respectively; $\mathbf{A}^{T}, \mathbf{A}^{*}, \mathbf{A}^{H}$, and $\mathbf{A}^{\dagger}$ denote the transpose, conjugate, Hermitian transpose and pseudo-inverse of $\mathbf{A}$, respectively; $[\mathbf{a}]_{m: n},[\mathbf{A}]_{m: n,:}$ denote the subvector of a from the $m$ th to the $n$th entries and the submatrix of $\mathbf{A}$ from the $m$ th to the $n$th rows, respectively; $\operatorname{Diag}(\mathbf{a})$ denotes the diagonal matrix formed by $\mathbf{a} ;\|\mathbf{a}\|_{0},\|\mathbf{a}\|_{2}$ and $\|\mathbf{A}\|_{F}$ denote the 0-norm, 2-norm of $\mathbf{a}$ and Frobenius norm of $\mathbf{A}$, respectively; $\otimes, \odot, *$ and $\circ$ denote the Kronecker, Khatri-Rao, Hadamard and outer products, respectively; $\mathcal{I}(m)$ denotes the set $\{1,2, \ldots, m\} ; \mathbf{0}_{m \times n}, \mathbf{I}_{m}$ and $\mathbf{e}_{n}^{(m)}$ denote the $m \times n$ zero matrix, the $m$ th-order identity matrix, and the $n$th column of $\mathbf{I}_{m}$ respectively; $\operatorname{range}(\mathbf{A}), \operatorname{ker}(\mathbf{A})$ and $\operatorname{dim}(\mathbf{A})$ denote the range, kernel subspaces and dimensionality of $\mathbf{A}$, respectively; $\operatorname{vec}(\cdot)$ denotes the vectorization operation; $C_{n}^{2} \triangleq n(n-1) / 2$, and $\mathbf{C}_{2}(\mathbf{A}) \in \mathbb{C}^{C_{m}^{2} \times C_{n}^{2}}$ denotes the 2nd compound matrix containing determinants of all $2 \times 2$ submatrices of $\mathbf{A} \in \mathbb{C}^{m \times n}$. In addition, all the relevant tensor definitions and lemmas in the text are presented in Appendix A.

\section{System Model}

We consider an IRS-assisted MIMO-OFDM system as illustrated in Fig. 1(a), where one base station (BS) equipped with $N_{\mathrm{B}}$ antennas and $M_{\mathrm{B}}$ RFCs communicates with $U$ singleantenna mobile stations (MS). The IRS is a programmable metasurface composed of $N_{\mathrm{I}}$ regularly arranged unit cells, forming a two-dimensional artificial structure [6]-[8]. The OFDM scheme occupies $K_{\mathrm{s}}$ subcarriers, where the carrier frequency and bandwidth are denoted by $f_{\mathrm{c}}$ and $f_{\mathrm{s}}$, respectively.

We suppose that one transmission frame contains $Q$ time slots, where the channel remains constant within each frame but varies across different frames. By assuming that the channel reciprocity holds, the uplink/downlink transmission within the $q$ th time slot of the $p$ th frame at the $k$ th subcarrier can be respectively expressed as

$$
\begin{aligned}
& \mathbf{y}_{p, q, k}^{\mathrm{ul}}=\mathbf{W}_{p, q, k}^{T} \mathbf{H}_{p, k}^{\mathrm{BI}} \boldsymbol{\Psi}_{p, q, k} \mathbf{H}_{p, k}^{\mathrm{IM}} \mathbf{s}_{p, q, k}^{\mathrm{ul}}+\mathbf{n}_{p, q, k}^{\mathrm{ul}}, \\
& \mathbf{y}_{p, q, k}^{\mathrm{dl}}=\left(\mathbf{H}_{p, k}^{\mathrm{IM}}\right)^{T} \boldsymbol{\Psi}_{p, q, k}\left(\mathbf{H}_{p, k}^{\mathrm{BI}}\right)^{T} \mathbf{F}_{p, q, k}^{\mathrm{dl}} \mathbf{s}_{p, q, k}^{\mathrm{dl}}+\mathbf{n}_{p, q, k}^{\mathrm{dl}},
\end{aligned}
$$

where $\mathbf{y}_{p, q, k}^{\mathrm{ul}}\left(\mathbf{n}_{p, q, k}^{\mathrm{ul}}\right) \in \mathbb{C}^{M_{\mathrm{B}}}, \mathbf{y}_{p, q, k}^{\mathrm{dl}}\left(\mathbf{n}_{p, q, k}^{\mathrm{dl}}\right) \in \mathbb{C}^{U}$ denote the uplink and donwlink received signals (noise), respectively; $\mathbf{s}_{p, q, k}^{\mathrm{ul}} \in \mathbb{C}^{U}, \mathbf{s}_{p, q, k}^{\mathrm{dl}} \in \mathbb{C}^{M_{\mathrm{B}}}$ denote the transmitted symbols. Also, $\mathbf{F}_{p, q, k}, \mathbf{W}_{p, q, k} \in \mathbb{C}^{N_{\mathrm{B}} \times M_{\mathrm{B}}}$ denote the precoding and combining beamformer, respectively, whilst $\boldsymbol{\Psi}_{p, q, k} \triangleq$ $\operatorname{Diag}\left(\boldsymbol{\psi}_{p, q, k}\right) \in \mathbb{C}^{N_{\mathrm{I}} \times N_{\mathrm{I}}}$ denotes the IRS coefficients, where 


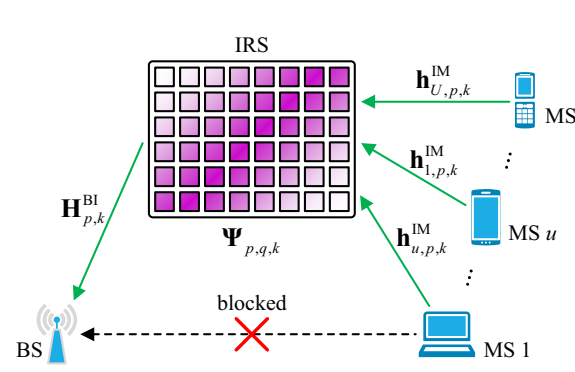

(a) Uplink MS-to-IRS-to-BS transmission.

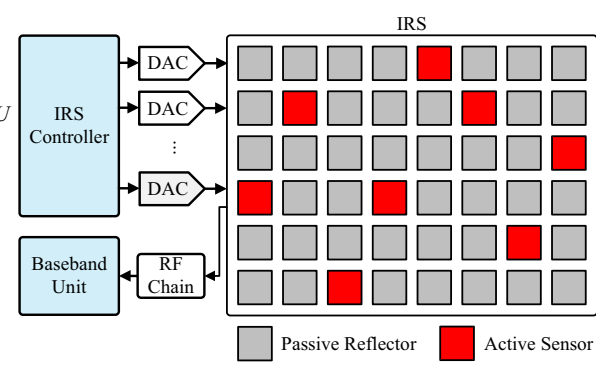

(b) Hybrid passive/active IRS structure.

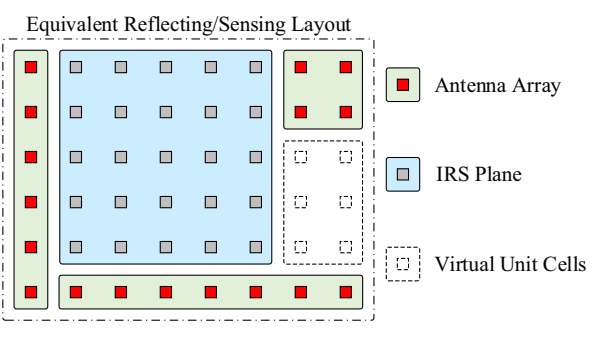

(c) Equivalent reflecting/sensing layout.

Fig. 1. An IRS-assisted mmWave MIMO-OFDM system. (a) The $U$ MSs transmit signals through the MS-to-IRS channels $\left\{\mathbf{h}_{p, k}^{\mathrm{IM}}\right\}_{u=1}^{U}$ to the IRS, which are reflected to the BS through the IRS-to-BS channel $\mathbf{H}_{p, k}^{\mathrm{BI}}$ with controlled phase shifts $\boldsymbol{\Psi}_{p, q, k}$. (b) The passive reflectors reflect signal waves with phase shifts designed by the IRS controller; the active sensors transfer training signals to baseband units. (c) The equivalent reflecting/sensing layout of the hybrid IRS architecture is formulated by reflector and antenna modules with virtual entries occupying the module spacing and irregular areas.

$\left[\boldsymbol{\psi}_{p, q, k}\right]_{n}=\eta_{n, p, q, k} e^{j \psi_{n, p, q, k}}$ with $\eta_{n, p, q, k} \in[0,1]$ and $\psi_{n, p, q, k} \in[0,2 \pi][31],[32] ; \mathbf{H}_{p, k}^{\mathrm{BI}} \in \mathbb{C}^{N_{\mathrm{B}} \times N_{\mathrm{I}}}$ and $\mathbf{H}_{p, k}^{\mathrm{IM}}=$ $\left[\mathbf{h}_{1, p, k}^{\mathrm{IM}}, \ldots, \mathbf{h}_{U, p, k}^{\mathrm{IM}}\right] \in \mathbb{C}^{N_{\mathrm{I}} \times U}$ denote the IRS-to-BS and MSto-IRS channels, respectively.

Many existing works, e.g., [14]-[21], seek to estimate the cascaded BS-IRS-MS channel from (1). This approach, however, experiences the following problems: (i) The training signal suffers from exacerbated propagation loss along the two-hop cascaded channel at higher frequencies; (ii) The required training time of cascaded channel estimation is proportional to the number of IRS units, such that large-scale IRSs with massive passive elements will result in extremely heavy training overhead; (iii) The most serious problem is that there inherently exist unavoidable ambiguities in the cascaded channel estimates, i.e., for any nonsingular diagonal $\boldsymbol{\Upsilon} \in \mathbb{C}^{N_{\mathrm{I}} \times N_{\mathrm{I}}}$, the scaled channels $\mathbf{H}_{p, k}^{\mathrm{BI}} \boldsymbol{\Upsilon}, \boldsymbol{\Upsilon}^{-1} \mathbf{H}_{p, k}^{\mathrm{IM}}$ do not affect the received signal $\mathbf{y}_{p, k}^{\mathrm{dl}(\mathrm{ul})}$. In multiuser communications, training ambiguities from multiple users lead to inaccurate estimates of the BS-IRS channel. Moreover, these estimation uncertainties will undermine the integration of IRS in many wireless services. For example, the IRS-based modulation of [33] can only broadcast properly modulated symbols to the receivers after equalizing the distortion effects of the transmitterIRS channel; the environment mapping of [34] manages to determine the object surfaces and scatter points based on exact measurements of the received signal strength (RSS), time of arrival (ToA) and angle of arrival/departure (AoA/AoD); the user localization and the mobility tracking of [35], [36] also require precise estimation of the propagation distance and azimuth/elevation angles from the MSs. Unfortunately, without any a priori information, the one-hop channels, as well as, the path parameters cannot be properly recovered from the cascaded channel estimation.

In order to overcome these shortcomings, we develop a hybrid IRS architecture composed of $N_{\mathrm{P}}$ passive reflectors and $N_{\mathrm{A}}$ active sensors to simultaneously reflect the signal waves and sense the one-hop channels, as illustrated in Fig. 1(b). The IRS-assisted system is able to directly sense the one-hop channels by observing the training signal at the IRS end. This design can also be equivalently viewed as a hybrid layout consisting of one or multiple IRS planes and a few

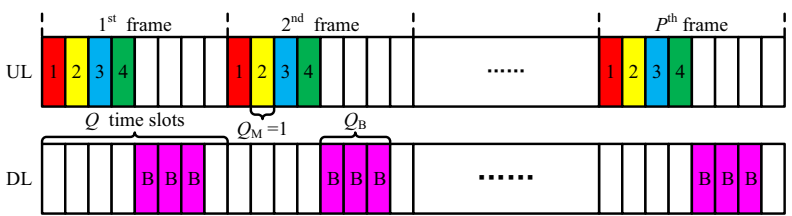

Fig. 2. Diagram of the training pilot allocation during $P$ frames. Each MS sends pilots in $Q_{\mathrm{M}}=1$ time slot of each frame; the BS sends pilots in $Q_{\mathrm{B}} \geq 2$ time slots of each frame. Pilots at different subcarriers can be arranged in identical time slots.

antenna nodes/arrays, which are located closely (relative to the signal wavelength) to formulate a regular $N_{\mathrm{x}} \times N_{\mathrm{y}}$-planar topology as illustrated in Fig. 1(c). For far-field propagation, the active/passive modules can be assumed to share wireless channels with identical multiple parameters. ${ }^{2}$ Since the passive entries do not contribute to the training signals received by the sensors, the number of reflectors supported by a given amount of active sensors can be theoretically extended without limitations, and virtual unit cells with no circuit can be employed to fill the module spacing and occupy the irregular areas of the layout topology. ${ }^{3}$ The proposed design can support hundreds or even thousands of IRS elements with $N_{\mathrm{A}}$ RFCs, achieving superior spectral and energy efficiency than an $N_{\mathrm{A}^{-}}$antenna relay [6], [37], [38]. We note that [22] also deploys up to two single-antenna anchors, such as controllers or idle users, near the IRS to assist the cascaded channel estimation. These anchors can help separate and recover the constant channels up to element-wise \pm sign uncertainty via multiple training steps with a total training overhead proportional to the size of IRS and the number of users. By deploying more sensing antennas around the IRS, our method is able to precisely recover the dynamic channels, as well as, the multipath parameters with less training overhead independent of $N_{\mathrm{I}}, N_{\mathrm{B}}$ or $U$.

As illustrated in Fig. 2, the uplink/downlink training occu-

\footnotetext{
${ }^{2}$ If the antenna arrays are placed further away from the IRS, the multipath parameters of channels experienced by the passive/active blocks may be different. Then, the sensor modules become anchors near the IRS as in [22].

${ }^{3}$ The proposed scheme offers considerable design freedom of the hybrid IRS structure or the equivalent layout. The reflectors and antennas can be arranged on identical or different substrate boards and individually implemented by existing printed circuit board techniques. They are only required to jointly formulate a regular planar topology along with virtual entries.
} 
pies $P$ frames, where the $u$ th MS sends $x_{u, p, k}$ in $Q_{\mathrm{M}}=1$ time slot of the $p$ th frame; the BS periodically sends precoded pilots $\left\{\mathbf{F}_{q, k} \mathbf{x}_{q, k}\right\}_{q=1}^{Q_{\mathrm{B}}}$ in $Q_{\mathrm{B}} \geq 2$ time slots of each frame. The training signals partially observed at the IRS are expressed as

$$
\begin{aligned}
& \mathbf{z}_{u, p, k}^{\mathrm{ul}}=\mathbf{w} * \mathbf{y}_{u, p, k}^{\mathrm{ul}}=\mathbf{w} *\left(\mathbf{h}_{u, p, k}^{\mathrm{IM}} x_{u, p, k}\right)+\mathbf{w} * \mathbf{n}_{u, p, k}^{\mathrm{ul}}, \\
& \mathbf{z}_{p, q, k}^{\mathrm{dl}}=\mathbf{w} * \mathbf{y}_{p, q, k}^{\mathrm{dl}}=\mathbf{w} *\left(\left(\mathbf{H}_{p, k}^{\mathrm{BI}}\right)^{T} \mathbf{F}_{q, k} \mathbf{x}_{q, k}\right)+\mathbf{w} * \mathbf{n}_{p, q, k}^{\mathrm{dl}},
\end{aligned}
$$

where $\mathbf{y}_{u, p, k}^{\mathrm{ul}}, \mathbf{y}_{p, q, k}^{\mathrm{dl}} \in \mathbb{C}^{N_{\mathrm{I}}}$ denote the arrived pilot signals; $\mathbf{z}_{u, p, k}^{\mathrm{ul}}, \mathbf{z}_{p, q, k}^{\mathrm{dl}} \in \mathbb{C}^{N_{\mathrm{I}}}$ denote the observed incomplete data; $\mathbf{n}_{u, p, k}^{\text {ul }}, \mathbf{n}_{p, q, k}^{\mathrm{dl}} \in \mathbb{C}^{N_{\mathrm{I}}}$ are the noise vectors. Also, w $\in\{0,1\}^{N_{\mathrm{I}}}$ is a binary indicator, where " $0 / 1$ " indicates the passive/active entry state. The Hadamard products represent the "filtering" process of effective signals by the hybrid layout.

We consider IRS-assisted systems working at the mmWave frequencies. By leveraging the sparse scattering feature of high-frequency waves, $\mathbf{H}_{p, k}^{\mathrm{BI}}$ and $\left\{\mathbf{h}_{u, p, k}^{\mathrm{IM}}\right\}_{u=1}^{U}$ can be characterized by the Saleh-Valenzuela model with $L_{0}$ and $\left\{L_{u}\right\}_{u=1}^{U}$ signal propagation paths respectively as ${ }^{4}$

$$
\begin{array}{r}
\mathbf{H}_{p, k}^{\mathrm{BI}}=\sum_{\ell=1}^{L_{0}} \underbrace{\alpha_{\ell, p} e^{-j 2 \pi \frac{(k-1) f_{\mathrm{s}} \tau_{0, \ell}}{K_{\mathrm{s}}}}}_{\alpha_{\ell, p, k}} \mathbf{a}_{\mathrm{B}}\left(\phi_{\mathrm{B}, \ell}, \theta_{\mathrm{B}, \ell}\right) \mathbf{a}_{\mathrm{I}}^{T}\left(\phi_{\mathrm{I}, \ell}, \theta_{\mathrm{I}, \ell}\right), \\
\mathbf{h}_{u, p, k}^{\mathrm{IM}}=\sum_{\ell=1}^{L_{u}} \underbrace{\beta_{u, \ell, p} e^{-j 2 \pi \frac{(k-1) f_{\mathrm{s}} \tau_{u, \ell}}{K_{\mathrm{s}}}}}_{\beta_{u, \ell, p, k}} \mathbf{a}_{\mathrm{I}}\left(\phi_{u, \ell}, \theta_{u, \ell}\right), u \in \mathcal{I}(U),
\end{array}
$$

where $\alpha_{\ell, p}, \beta_{u, \ell, p}$ denote the complex path gains in the $p$ th frame; $\tau_{0, \ell}, \tau_{u, \ell}$ denote the propagation time delays; $\phi_{\mathrm{B}, \ell}\left(\theta_{\mathrm{B}, \ell}\right), \phi_{\mathrm{I}, \ell}\left(\theta_{\mathrm{I}, \ell}\right)$ denote the azimuth (elevation) AoA and AoD of the IRS-to-BS paths, respectively; $\phi_{u, \ell}\left(\theta_{u, \ell}\right)$ denotes the azimuth (elevation) AoA at the IRS from the $u$ th MS. The array response vectors of the BS and IRS are denoted by $\mathbf{a}_{\mathrm{B}}(\phi, \theta) \in \mathbb{C}^{N_{\mathrm{B}}}$ and $\mathbf{a}_{\mathrm{I}}(\phi, \theta) \in \mathbb{C}^{N_{\mathrm{I}}}$, respectively. The IRS elements are assumed to form an uniform planar array (UPA), while the BS can employ either a UPA or a uniform linear array (ULA) of antennas. We represent the response vector as $\mathbf{a}_{\mathrm{I}}(\phi, \theta) \triangleq \mathbf{a}_{\mathbf{x}}(\phi, \theta) \otimes \mathbf{a}_{\mathrm{y}}(\phi, \theta)$, where

$\mathbf{a}_{\mathrm{x}}(\phi, \theta)=\left[1, e^{j 2 \pi \frac{d}{\lambda_{\mathrm{c}}} \sin \phi \cos \theta}, \ldots, e^{j 2 \pi \frac{d}{\lambda_{\mathrm{c}}}\left(N_{\mathrm{x}}-1\right) \sin \phi \cos \theta}\right]^{T}$,

$\mathbf{a}_{\mathrm{y}}(\phi, \theta)=\left[1, e^{j 2 \pi \frac{d}{\lambda_{\mathrm{c}}} \sin \phi \sin \theta}, \ldots, e^{j 2 \pi \frac{d}{\lambda_{\mathrm{c}}}\left(N_{\mathrm{y}}-1\right) \sin \phi \sin \theta}\right]^{T}$,

denote the azimuth and elevation array response vectors, respectively, whilst $d$ and $\lambda_{\mathrm{c}}$ denote the adjacent spacing and wavelength, respectively. ${ }^{5}$ Based on (3), (4), the cascaded channel in (1) can be equivalently viewed as a channel with

\footnotetext{
${ }^{4} \mathrm{We}$ assume that the path angles and delays remain constant, while the path gains vary across different frames [39], [40].

${ }^{5}$ The steering vectors only determine the operation of angle parameter recovery. The proposed tensor completion scheme can apply to arbitrary $2 \mathrm{D}$ layout with array responses $\mathbf{a}_{\mathrm{I}}(\cdot)=\mathbf{a}_{\mathrm{x}}(\cdot) \otimes \mathbf{a}_{\mathrm{y}}(\cdot)$.
}

$L_{0} L_{u}$ scattering paths. One can verify that the traditional cascaded channel estimation methods cannot recover precise path parameters but only $L_{0} L_{u}$ sets of equivalent parameters, i.e., $\sin \phi_{\mathrm{I}, \ell_{0}} \cos \theta_{\mathrm{I}, \ell_{0}}+\sin \phi_{u, \ell_{u}} \cos \theta_{u, \ell_{u}}, \sin \phi_{\mathrm{I}, \ell_{0}} \sin \theta_{\mathrm{I}, \ell_{0}}+$ $\sin \phi_{u, \ell_{u}} \sin \theta_{u, \ell_{u}}, \tau_{0, \ell_{0}}+\tau_{u, \ell_{u}}$ and $\alpha_{\ell_{0}, p} \beta_{u, \ell_{u}, p}, \forall \ell_{0} \in$ $\mathcal{I}\left(L_{0}\right), \ell_{u} \in \mathcal{I}\left(L_{u}\right)$ [16], [36].

Combining (2)-(4), we can rewrite the partially observed training signals as

$$
\begin{aligned}
\mathbf{z}_{u, p, k}^{\mathrm{ul}=} & \mathbf{w} *\left(\sum_{\ell=1}^{L_{u}} \beta_{u, \ell, p, k} \mathbf{a}_{\mathrm{I}}\left(\phi_{u, \ell}, \theta_{u, \ell}\right) x_{u, p, k}\right)+\mathbf{w} * \mathbf{n}_{u, p, k}^{\mathrm{ul}} \\
= & \mathbf{w} *\left(\mathbf{A}_{\mathbf{x}, u}^{\mathrm{ul}} \odot \mathbf{A}_{\mathrm{y}, u}^{\mathrm{ul}}\right) \mathbf{g}_{u, p, k}^{\mathrm{ul}}+\mathbf{w} * \mathbf{n}_{u, p, k}^{\mathrm{ul}}, \\
\mathbf{z}_{p, q, k}^{\mathrm{dl}}= & \mathbf{w} *\left(\sum_{\ell=1}^{L_{0}} \alpha_{\ell, p, k} \mathbf{a}_{\mathrm{I}}\left(\phi_{\mathrm{I}, \ell}, \theta_{\mathrm{I}, \ell}\right) \mathbf{a}_{\mathrm{B}}^{T}\left(\phi_{\mathrm{B}, \ell}, \theta_{\mathrm{B}, \ell}\right) \mathbf{F}_{q, k} \mathbf{x}_{q, k}\right) \\
& +\mathbf{w} * \mathbf{n}_{p, q, k}^{\mathrm{dl}} \\
= & \mathbf{w} *\left(\mathbf{A}_{\mathbf{x}}^{\mathrm{dl}} \odot \mathbf{A}_{\mathrm{y}}^{\mathrm{dl}}\right) \mathbf{g}_{p, q, k}^{\mathrm{dl}}+\mathbf{w} * \mathbf{n}_{p, q, k}^{\mathrm{dl}},
\end{aligned}
$$

where $\mathbf{A}_{\mathrm{x}(\mathrm{y}), u}^{\mathrm{ul}} \in \mathbb{C}^{N_{\mathrm{x}(\mathrm{y})} \times L_{u}}$ collects the steering vectors $\left\{\mathbf{a}_{\mathrm{x}(\mathrm{y})}\left(\phi_{u, \ell}, \theta_{u, \ell}\right)\right\} ; \mathbf{g}_{u, p, k}^{\mathrm{ul}} \in \mathbb{C}^{L_{u}}$ contains the equivalent gains $\left\{\beta_{u, \ell, p, k} x_{u, p, k}\right\}$. Also, $\mathbf{A}_{\mathrm{x}(\mathrm{y})}^{\mathrm{dl}} \in \mathbb{C}^{N_{\mathrm{x}(\mathrm{y})} \times L_{0}}$ and $\mathbf{g}_{p, q, k}^{\mathrm{ul}} \in \mathbb{C}^{L_{0}}$ are similarly defined. In the following sections, we will leverage the concept of tensor completion to address the fundamental channel estimation problem by recovering (5).

\section{TENSOR COMPLETION-BASED NARROWBAND CHANNEL ESTIMATION}

We first consider a particular training mode such that the pilot symbols are arranged on a single subcarrier, i.e., $k=1$. This mode is compatible with narrowband systems, which can be extended to the wideband OFDM systems. By concatenating $P$ and $Q_{\mathrm{B}} P$ training time slots of (5a), (5b) respectively, we can derive ${ }^{6}$

$$
\begin{aligned}
& \mathbf{Z}^{\mathrm{ul}}=\mathbf{W}^{\mathrm{ul}} * \mathbf{Y}^{\mathrm{ul}}=\mathbf{W}^{\mathrm{ul}} *\left(\left(\mathbf{A}_{\mathrm{x}}^{\mathrm{ul}} \odot \mathbf{A}_{\mathrm{y}}^{\mathrm{ul}}\right) \mathbf{G}_{\beta}^{T}+\mathbf{N}^{\mathrm{ul}}\right), \\
& \mathbf{Z}^{\mathrm{dl}}=\mathbf{W}^{\mathrm{dl}} * \mathbf{Y}^{\mathrm{dl}}=\mathbf{W}^{\mathrm{dl}} *\left(\left(\mathbf{A}_{\mathrm{x}}^{\mathrm{dl}} \odot \mathbf{A}_{\mathrm{y}}^{\mathrm{dl}}\right) \mathbf{G}_{\alpha}^{T}+\mathbf{N}^{\mathrm{dl}}\right),
\end{aligned}
$$

where $\mathbf{W}^{\mathrm{ul}} \triangleq \mathbf{w} \otimes \mathbf{1}_{1 \times P}, \mathbf{W}^{\mathrm{dl}} \triangleq \mathbf{w} \otimes \mathbf{1}_{1 \times Q_{\mathrm{B}} P}$ denote the indicators; $\mathbf{G}_{\beta} \in \mathbb{C}^{P \times L}, \mathbf{G}_{\alpha} \in \mathbb{C}^{Q_{\mathrm{B}} P \times L_{0}}$ denote the equivalent gains, where $\left[\mathbf{G}_{\beta}\right]_{p, \ell}=x_{p} \beta_{\ell, p},\left[\mathbf{G}_{\alpha}\right]_{(q-1) P+p, \ell}=$ $\mathbf{x}_{q}^{T} \mathbf{F}_{q}^{T} \mathbf{a}_{\mathrm{B}}\left(\phi_{\mathrm{B}, \ell}, \theta_{\mathrm{B}, \ell}\right) \alpha_{\ell, p}$. Since (6a), (6b) are in similar forms, we mainly focus on the uplink channel estimation, whose solution similarly applies to the downlink case. ${ }^{7}$

\section{A. Space-Time Signal Tensor}

According to (41), $\mathbf{Y}(\mathbf{Z})$ in (6a) can be regarded as a tensor matricization, i.e., $\operatorname{Matr}(\mathcal{Y}(\mathcal{Z}) ;[2,1], 3)$, where

$$
\begin{aligned}
& \mathcal{Y}=\sum_{\ell=1}^{L} \mathbf{a}_{\mathrm{x}, \ell} \circ \mathbf{a}_{\mathrm{y}, \ell} \circ \mathbf{g}_{\beta, \ell}+\mathcal{N}=\llbracket \mathbf{a}_{\mathrm{x}}, \mathbf{a}_{\mathrm{y}}, \mathbf{G}_{\beta} \rrbracket+\mathcal{N}, \\
& \mathcal{Z}=\mathcal{W} * \mathcal{Y}=\mathcal{W} * \sum_{\ell=1}^{L} \mathbf{a}_{\mathrm{x}, \ell} \circ \mathbf{a}_{\mathrm{y}, \ell} \circ \mathbf{g}_{\beta, \ell}+\mathcal{W} * \mathcal{N},
\end{aligned}
$$

\footnotetext{
${ }^{6}$ The subscripts " $u$ ", " $k$ " are temporally omitted for notational simplicity.

${ }^{7}$ The superscript "ul" is omitted hereinafter. All the following variables correspond to the uplink case unless otherwise stated.
} 
with $\mathbf{a}_{\mathrm{x}, \ell} \triangleq \mathbf{a}_{\mathrm{x}}\left(\phi_{\ell}, \theta_{\ell}\right), \mathbf{a}_{\mathrm{y}, \ell} \triangleq \mathbf{a}_{\mathrm{y}}\left(\phi_{\ell}, \theta_{\ell}\right), \mathbf{g}_{\beta, \ell} \triangleq\left[\mathbf{G}_{\beta}\right]_{:, \ell}$; $\mathcal{W} \in\{0,1\}^{N_{\mathrm{x}} \times N_{\mathrm{y}} \times P}$ and $\mathcal{N} \in\{0,1\}^{N_{\mathrm{x}} \times N_{\mathrm{y}} \times P}$ are the tensorial forms of $\mathbf{W} \triangleq \mathbf{W} \otimes \mathbf{1}_{1 \times P}$ and $\mathbf{N}$, respectively. Recovering the signal tensor $\mathcal{Y}$, or equivalently its CPD factors $\left\{\mathbf{a}_{\mathrm{x}, \ell}, \mathbf{a}_{\mathrm{y}, \ell}, \mathbf{g}_{\beta, \ell}\right\}_{\ell=1}^{L}$, from the observation $\mathcal{Z}$ is referred to as a tensor completion problem [28]-[30]. These existing methods, however, resort to iterative operations with empirical termination threshold, yielding unpredictable overhead and complexity. They were designed to deal with discretely sampled tensors, and cannot efficiently handle data with a few regularly sampled entries. Moreover, they are not supported by theoretical uniqueness analysis, and they are not guaranteed to return the exact solutions. In order to obtain a straightforward solver as well as an intuitive guidance of hybrid reflecting/sensing design and training resource allocation, we need to fully exploit the distribution pattern and structural information of the training data.

We note that the $(i, j)$ th passive entry leads to an all-zero frontal (mode-3) fiber $\mathbf{w}_{i, j,:}=\mathbf{0}_{P \times 1}$, which filters out the corresponding signal $\mathbf{y}_{i, j,:} \in \mathbb{C}^{P}$ of $\mathcal{Y}$. Therefore, solving (7) is referred to as a fiber sampling tensor completion problem [41], [42]. It can be verified that the $(i, j)$ th all-zero fiber of $\mathcal{W}$ corresponds to the $\left((i-1) N_{\mathrm{y}}+j\right)$ th all-zero row of $\mathbf{W}$. Then, one can obtain an effective observed signal submatrix $\mathbf{Z}_{\text {sub }} \in \mathbb{C}^{N_{\mathrm{A}} \times P}$ as

$$
\mathbf{Z}_{\text {sub }}=\mathbf{S}_{\mathbf{W}} \mathbf{Y}=\boldsymbol{\Xi}\left(\mathbf{A}_{\mathbf{x}}, \mathbf{A}_{\mathrm{y}}\right) \mathbf{G}_{\beta}^{T}+\mathbf{S}_{\mathbf{W}} \mathbf{N}
$$

where $\mathbf{S}_{\mathbf{W}} \in\{0,1\}^{N_{\mathrm{A}} \times N_{\mathrm{I}}}$ is a row-selection matrix that selects the $N_{\mathrm{A}}$ rows of $\mathbf{Y}$ that have not been zeroed by $\mathbf{W}$; $\boldsymbol{\Xi}\left(\mathbf{A}_{\mathrm{x}}, \mathbf{A}_{\mathrm{y}}\right) \triangleq \mathbf{S}_{\mathbf{W}}\left(\mathbf{A}_{\mathrm{x}} \odot \mathbf{A}_{\mathrm{y}}\right) \in \mathbb{C}^{N_{\mathrm{A}} \times L}$.

\section{B. Uniqueness Condition of Tensor Completion}

In order to accurately recover the signal tensor, as well as, the channel parameters, it is necessary to explore the uniqueness condition of CPD tensor completion with fiber sampling. ${ }^{8}$

We define a binary diagonal matrix $\mathbf{D}_{\text {sel }}=\operatorname{Diag}\left(\mathbf{d}_{\text {sel }}\right) \in$ $\{0,1\}{ }^{C_{N_{\mathrm{x}}}^{2} C_{N_{\mathrm{y}}}^{2} \times C_{N_{\mathrm{x}}}^{2} C_{N_{\mathrm{y}}}^{2}}$ with $\mathbf{d}_{\text {sel }} \in\{0,1\}{ }^{C_{N_{\mathrm{x}}}^{2} C_{N_{\mathrm{y}}}^{2}}$ lexicographically defined as

$$
\begin{gathered}
\mathbf{d}_{\mathrm{sel}}=\left[d_{(1,2),(1,2)}, d_{(1,2),(1,3)},\right. \\
\left.\ldots, d_{\left(N_{\mathrm{x}}-1, N_{\mathrm{x}}\right),\left(N_{\mathrm{y}}-1, N_{\mathrm{y}}\right)}\right]^{T} \\
d_{\left(i_{1}, i_{2}\right),\left(j_{1}, j_{2}\right)}=\left\{\begin{array}{c}
1, \text { if } \mathbf{y}_{i_{1}, j_{1},:}, \mathbf{y}_{i_{1}, j_{2},:}, \mathbf{y}_{i_{2}, j_{1},:}, \mathbf{y}_{i_{2}, j_{2},} \\
\text { are observable fibers } \\
0, \text { otherwise }
\end{array}\right.
\end{gathered}
$$

where $1 \leq i_{1}<i_{2} \leq N_{\mathrm{x}}, 1 \leq j_{1}<j_{2} \leq N_{\mathrm{y}}$. Physically, $d_{\left(i_{1}, i_{2}\right),\left(j_{1}, j_{2}\right)}$ indicates whether the corresponding $2 \times 2$ element submatrix of the hybrid layout can observe the complete effective signals. We define a matrix $\mathbf{P}^{(\ell)} \in \mathbb{C}^{N_{\mathrm{x}} \times N_{\mathrm{y}}}$ with indeterminate entries as

$$
p_{i j}^{(\ell)}= \begin{cases}{\left[\mathbf{a}_{\mathrm{x}, \ell} \otimes \mathbf{a}_{\mathrm{y}, \ell}\right]_{(i-1) N_{\mathrm{y}}+j},} & \text { if } \mathbf{w}_{i, j,:}=\mathbf{1}_{P \times 1}, \\ \text { indeterminate, } & \text { if } \mathbf{w}_{i, j,:}=\mathbf{0}_{P \times 1} .\end{cases}
$$

\footnotetext{
${ }^{8}$ All the theoretical analysis presented in the following content applies to noiseless signals. That is, for the sake of clarify, the noise terms in the received signals are temporarily omitted unless otherwise specified.
}

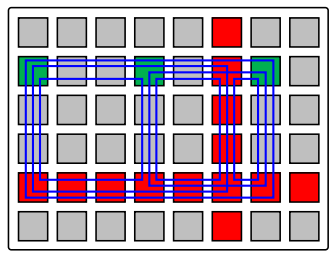

(a) Suboptimal configuration

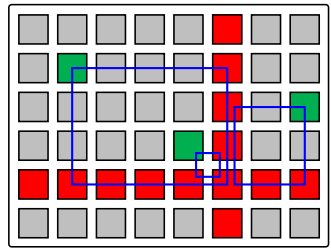

(b) Worst configuration
Fig. 3. Diagram of specific hybrid IRS designs. The gray squares denote the passive entries; the red squares denote the basic active sensors ensuring the graph connectivity; the green squares denote the extra active sensors contributing to $\left\|\mathbf{d}_{\text {sel }}\right\|_{0}$; the blue wireframes mark the effective $2 \times 2$ submatrices. (a) Suboptimal distribution, where $N$ extra active entries yield $\left\|\mathbf{d}_{\text {sel }}\right\|_{0}=C_{N+1}^{2}$. (b) Worst distribution, where $N$ extra active sensors yield $\left\|\mathbf{d}_{\mathrm{sel}}\right\|_{0}=N$.

By leveraging the graph theory, this incomplete matrix $\mathbf{P}^{(\ell)}$ can be related to a bipartite graph denoted by $\mathbb{P}^{(\ell)}$, whose two groups of vertices are $\mathcal{I}\left(N_{\mathrm{x}}\right)$ and $\mathcal{I}\left(N_{\mathrm{y}}\right)$. Now, $\mathcal{P}^{(\ell)}$ denotes the edge set, or supports, associated with $\mathbb{P}^{(\ell)}$, where the edge weight of $(i, j) \in \mathcal{P}^{(\ell)}$ is $p_{i j}^{(\ell)}$. We derive a restricted bipartite graph $\widetilde{\mathbb{P}}^{(\ell)}$ with an edge set $\widetilde{\mathcal{P}}^{(\ell)}=\left\{(i, j) \in \mathcal{P}^{(\ell)} \mid p_{i j}^{(\ell)} \neq 0\right\}$. Now, a uniqueness condition of fiber sampling CPD-tensor completion is presented as follows:

Theorem 1 [41]. Consider a tensor $\mathcal{Y} \in \mathbb{C}^{N_{\mathrm{x}} \times N_{\mathrm{y}} \times P}$ with factor matrices $\left\{\mathbf{A}_{\mathrm{x}}, \mathbf{A}_{\mathrm{y}}, \mathbf{G}_{\beta}\right\}$, partially observed by $\mathcal{Z}$ in (7). Let $\mathbf{D}_{\text {sel }}=\operatorname{Diag}\left(\mathbf{d}_{\text {sel }}\right)$ as defined in (9), and let $\widetilde{\mathbb{P}}^{(\ell)}$ be the restricted bipartite graph of $\mathbf{P}^{(\ell)}$ as defined in (10). If

$$
\left\{\begin{array}{l}
\operatorname{rank}\left(\mathbf{G}_{\beta}\right)=L, \\
\operatorname{rank}\left(\mathbf{D}_{\text {sel }}\left(\mathbf{C}_{2}\left(\mathbf{A}_{\mathrm{x}}\right) \odot \mathbf{C}_{2}\left(\mathbf{A}_{\mathrm{y}}\right)\right)\right)=C_{L}^{2}, \\
\widetilde{\mathbb{P}}^{(\ell)} \text { is a connected graph, } \forall \ell \in \mathcal{I}(L),
\end{array}\right.
$$

then the rank of $\mathcal{Y}$ equals $L$, and its $\mathrm{CPD}$ is unique up to scaling and permutation ambiguity.

In the generic case, the first two conditions in (11) become $P \geq L$ and $\left\|\mathbf{d}_{\text {sel }}\right\|_{0} \geq C_{L}^{2}$, respectively. A necessary condition to ensure the graph connectivity of $\widetilde{\mathbb{P}}^{(\ell)}$ is that every row and column of $\mathbf{P}^{(\ell)}$ get sampled at least once with nonzero values. Physically, this condition indicates that the active sensing network, i.e., $\mathbf{W}_{:,:, p}$, cannot partition the received signal $\mathbf{a}_{\mathrm{x}, \ell} \mathbf{a}_{\mathrm{y}, \ell}^{T}$ into "disconnected" submatrices. In our system, the graphs $\left\{\mathbb{P}^{(\ell)}\right\}_{\ell=1}^{L}$ share the identical topology. Compared with the anchor-based method [22] with a training overhead of $2 N_{\mathrm{I}}+U+\max \left(U,\left\lceil\frac{U N_{\mathrm{I}}}{N_{\mathrm{B}}}\right\rceil\right)$, the proposed method reduces the overhead to the order of $L$.

Remark. According to the uniqueness analysis, one can arrange the active IRS entries to obtain as many effective $2 \times 2$ submatrices as possible, provided the graph connectivity is satisfied. As illustrated in Fig. 3, the basic configuration is to completely sample one row and one column of the IRS with $\left(N_{\mathrm{x}}+N_{\mathrm{y}}-1\right)$ active sensors. Fig. 3(a), (b) show a suboptimal and the worst sampling distribution cases of totally $\left(N+N_{\mathrm{x}}+\right.$ $N_{\mathrm{y}}-1$ ) active elements, which respectively provide $C_{N+1}^{2}$ and $N$ effective $2 \times 2$-submatrices. Therefore, the minimal number of active sensors that ensures the uniqueness condition is (a) $N_{\mathrm{A}} \geq N_{\mathrm{x}}+N_{\mathrm{y}}+L$; (b) $N_{\mathrm{A}} \geq N_{\mathrm{x}}+N_{\mathrm{y}}+C_{L}^{2}-1$.

We present in Table I the statistical mean of $\left\|\mathbf{d}_{\text {sel }}\right\|_{0}$ versus the number of randomly distributed extra sensors, i.e., 
$N_{\mathrm{A}}-\left(N_{\mathrm{x}}+N_{\mathrm{y}}-1\right)$. The table indicates that to estimate a sparse channel with 3-5 scattering paths, i.e., $C_{L+1}^{2} \leq 15$, $N_{\mathrm{A}} \geq N_{\mathrm{x}}+N_{\mathrm{y}}+7$ can statistically guarantee the feasibility of estimation; $N_{\mathrm{A}} \geq N_{\mathrm{x}}+N_{\mathrm{y}}+14$ can strictly ensure the validity of each training trial.

\section{Proposed Algorithm}

We need a preliminary step to determine the tensor rank if it is unknown. Given the effective signal $\mathbf{Z}_{\text {sub }}$ with i.i.d. Gaussian noise $\mathbf{S}_{\mathbf{W}} \mathbf{N}$ in (8), we adopt a minimum description length (MDL) method to estimate the number of signal path components $L$ [43]. We compute the eigenvalue decomposition of the approximated covariance matrix of $\mathbf{Z}_{\text {sub }}$ as

$$
\mathbf{\Sigma}_{\mathbf{Z}_{\mathrm{sub}}}=\frac{1}{N_{\mathrm{A}}} \mathbf{Z}_{\mathrm{sub}}^{H} \mathbf{Z}_{\mathrm{sub}}=\mathbf{V} \boldsymbol{\Lambda} \mathbf{V}^{H}
$$

where $\boldsymbol{\Lambda} \triangleq \operatorname{Diag}\left(\left[\lambda_{1}, \ldots, \lambda_{P}\right]^{T}\right)$ contains the eigenvalues in descending order; $\mathbf{V} \in \mathbb{C}^{P \times P}$ denotes the corresponding eigenvectors. The rank of $\mathbf{Z}_{\text {sub }}$ is estimated by the MDL criterion as

$$
\begin{aligned}
L=\arg \min _{\ell} & \frac{\ell}{2}(2 P-\ell) \log \left(N_{\mathrm{A}}\right) \\
& -N_{\mathrm{A}}(P-\ell) \log \left(\frac{\prod_{p=\ell+1}^{P} \lambda_{p}^{\frac{1}{P-\ell}}}{\frac{1}{P-\ell} \sum_{p=\ell+1}^{P} \lambda_{p}}\right) .
\end{aligned}
$$

According to [41], [44], we develop an algebraic approach to solve the problem (7). By capitalizing on the low-rank feature of $\mathbf{Z}$, we compress its effective part, i.e., $\mathbf{Z}_{\text {sub }}$ in (8), by the singular value decomposition (SVD) as

$$
\begin{aligned}
\mathbf{Z}_{\text {sub }} & =\mathbf{U}_{\text {sub }} \boldsymbol{\Sigma}_{\text {sub }} \mathbf{V}_{\text {sub }}^{H}, \overline{\mathbf{Z}} \triangleq \mathbf{S}_{\mathbf{W}}^{T} \mathbf{U}_{\text {sub }} \boldsymbol{\Sigma}_{\text {sub }}, \\
\overline{\mathcal{Z}} & =\operatorname{Tens}\left(\overline{\mathbf{Z}} ;\left[N_{\mathrm{x}}, N_{\mathrm{y}}, L\right],[2,1], 3\right)
\end{aligned}
$$

where $\mathbf{U}_{\text {sub }} \in \mathbb{C}^{N_{\mathrm{A}} \times L}, \boldsymbol{\Sigma}_{\text {sub }} \in \mathbb{C}^{L \times L}$ and $\mathbf{V}_{\text {sub }} \in \mathbb{C}^{P \times L}$; $\overline{\mathbf{Z}} \in \mathbb{C}^{N_{\mathrm{I}} \times L}$ and $\overline{\mathcal{Z}} \in \mathbb{C}^{N_{\mathrm{x}} \times N_{\mathrm{y}} \times L}$ denote the compressed signal matrix and tensor, respectively. When $\min \left(N_{\mathrm{A}}, P\right) \geq L$ holds, $\operatorname{range}\left(\mathbf{Z}_{\text {sub }}\right)=\operatorname{range}\left(\mathbf{U}_{\text {sub }} \boldsymbol{\Sigma}_{\text {sub }}\right)$, and there exists a nonsingular matrix $\mathbf{F} \in \mathbb{C}^{L \times L}$ such that

$$
\mathbf{U}_{\mathrm{sub}} \boldsymbol{\Sigma}_{\mathrm{sub}} \mathbf{F}^{-T}=\boldsymbol{\Xi}\left(\mathbf{A}_{\mathrm{x}}, \mathbf{A}_{\mathrm{y}}\right), \mathbf{V}_{\mathrm{sub}}^{*} \mathbf{F}=\mathbf{G}_{\beta} .
$$

We define $\mathbf{Q}_{2}(\overline{\mathcal{Z}}) \in \mathbb{C}^{C_{N_{\mathrm{x}}}^{2} C_{N_{\mathrm{y}}}^{2} \times C_{L+1}^{2}}$ as a tensor-to-matrix transform, whose $\ell$ th column is given as

$$
\operatorname{vec}\left(\mathbf{C}_{2}^{T}\left(\overline{\mathbf{Z}}_{:,:, \ell_{1}}+\overline{\mathbf{Z}}_{:,:, \ell_{2}}\right)-\mathbf{C}_{2}^{T}\left(\overline{\mathbf{Z}}_{:,:, \ell_{1}}\right)-\mathbf{C}_{2}^{T}\left(\overline{\mathbf{Z}}_{:,:, \ell_{2}}\right)\right),
$$

where $\ell \triangleq \frac{1}{2}\left(\ell_{1}-1\right)\left(2 L-\ell_{1}\right)+\ell_{2}, 1 \leq \ell_{1} \leq \ell_{2} \leq L$. Let $\mathbf{S}_{\text {sel }} \in\{0,1\}^{\left\|\mathbf{d}_{\text {sel }}\right\|_{0} \times C_{N_{\mathrm{x}}}^{2} C_{N_{\mathrm{y}}}^{2}}$ denote the row-selection matrix that selects the effective parts of $\mathbf{Q}_{2}(\overline{\mathcal{Z}})$ indicated by $\mathbf{d}_{\text {sel }}$. Then, the effective rows $\mathbf{S}_{\text {sel }} \mathbf{Q}_{2}(\overline{\mathcal{Z}})$ can be equivalently rewritten by invoking [44, Lemma $2.17,3.11]$ as

$$
\mathbf{S}_{\mathrm{sel}} \mathbf{Q}_{2}(\overline{\mathcal{Z}})=\mathbf{S}_{\mathrm{sel}}\left(\mathbf{C}_{2}\left(\mathbf{A}_{\mathrm{x}}\right) \odot \mathbf{C}_{2}\left(\mathbf{A}_{\mathrm{y}}\right)\right) \boldsymbol{\Omega}_{2}^{T}(\mathbf{F}) \mathbf{T}_{S},
$$

with $\boldsymbol{\Omega}_{2}(\mathbf{F}) \in \mathbb{C}^{L^{2} \times C_{L}^{2}}, \mathbf{T}_{S} \in \mathbb{C}^{L^{2} \times C_{L+1}^{2}}$ being defined as

$$
\begin{aligned}
\mathbf{\Omega}_{2}(\mathbf{F})=2\left[\pi_{S}\left(\mathbf{f}_{1}, \mathbf{f}_{2}\right), \pi_{S}\left(\mathbf{f}_{1}, \mathbf{f}_{3}\right), \ldots, \pi_{S}\left(\mathbf{f}_{L-1}, \mathbf{f}_{L}\right)\right] \\
\mathbf{T}_{S}=\left[\pi_{S}\left(\mathbf{e}_{1}^{(L)}, \mathbf{e}_{1}^{(L)}\right), \pi_{S}\left(\mathbf{e}_{1}^{(L)}, \mathbf{e}_{2}^{(L)}\right)\right. \\
\left.\ldots, \pi_{S}\left(\mathbf{e}_{L}^{(L)}, \mathbf{e}_{L}^{(L)}\right)\right]
\end{aligned}
$$

where $\pi_{S}(\mathbf{x}, \mathbf{y}) \triangleq \frac{1}{2}(\mathbf{x} \otimes \mathbf{y}+\mathbf{y} \otimes \mathbf{x})$ is the symmetrization mapping of $\operatorname{vec}\left(\mathbf{y} \mathbf{x}^{T}\right) ; \mathbf{T}_{S}$ denotes an orthogonal basis set of range $\left(\pi_{S}\right)$ with $\left\{\mathbf{e}_{\ell_{1}}^{(L)} \otimes \mathbf{e}_{\ell_{2}}^{(L)}\right\}_{1 \leq \ell_{1} \leq \ell_{2} \leq L}$ being a canonical basis of $\mathbb{C}^{L^{2}}$. By combining (17), (18), we derive the following subspace relationship [44, Proposition 2.13]:

$$
\begin{aligned}
\mathbf{T}_{S} \operatorname{ker}\left(\mathbf{S}_{\text {sel }} \mathbf{Q}_{2}(\overline{\mathcal{Z}})\right) & =\operatorname{ker}\left(\boldsymbol{\Omega}_{2}^{T}(\mathbf{F})\right) \cap \operatorname{range}\left(\pi_{S}\right) \\
& =\operatorname{range}\left(\mathbf{F}^{-T} \odot \mathbf{F}^{-T}\right) .
\end{aligned}
$$

We denote the basis of the $L$-dimensional subspace (19) by $\mathbf{M}=\left[\mathbf{m}_{1}, \ldots, \mathbf{m}_{L}\right] \in \mathbb{C}^{L^{2} \times L}$. If $\mathbf{S}_{\text {sel }}\left(\mathbf{C}_{2}\left(\mathbf{A}_{\mathrm{x}}\right) \odot \mathbf{C}_{2}\left(\mathbf{A}_{\mathrm{y}}\right)\right)$ in (17) has full rank, there exists a nonsingular $\mathbf{B} \in \mathbb{C}^{L \times L}$ such that

$$
\begin{aligned}
\mathbf{M} & =\left(\mathbf{F}^{-T} \odot \mathbf{F}^{-T}\right) \mathbf{B}^{T}, \\
\mathcal{M} & =\operatorname{Tens}(\mathbf{M} ;[L, L, L],[2,1], 3)=\llbracket \mathbf{F}^{-T}, \mathbf{F}^{-T}, \mathbf{B} \rrbracket .
\end{aligned}
$$

One can solve the CPD $\mathcal{M} \in \mathbb{C}^{L \times L \times L}$ in (20) by the generalized eigenvalue decomposition (GEVD) method [44], i.e., $\mathbf{M}_{:,:, \ell_{1}} \mathbf{F D i a g}\left([\mathbf{B}]_{\ell_{2},:}^{T}\right)=\mathbf{M}_{:,:, \ell_{2}} \mathbf{F D i a g}\left([\mathbf{B}]_{\ell_{1},:}^{T}\right), 1 \leq \ell_{1} \neq$ $\ell_{2} \leq L$. Once the nonsingular $\mathbf{F}$ has been derived, one can directly compute $\boldsymbol{\Xi}\left(\mathbf{A}_{\mathrm{x}}, \mathbf{A}_{\mathrm{y}}\right)$ and $\mathbf{G}_{\beta}$ by (15).

Now, the remaining problem is to determine $\mathbf{A}_{\mathrm{x}}, \mathbf{A}_{\mathrm{y}}$ given a partial observation $\boldsymbol{\Xi}\left(\mathbf{A}_{\mathrm{x}}, \mathbf{A}_{\mathrm{y}}\right)$. Recall that $\mathbf{P}^{(\ell)}$ in (10) can be obtained by reshape $\left(\mathbf{S}_{\mathbf{W}}^{T}\left[\boldsymbol{\Xi}\left(\mathbf{A}_{\mathrm{x}}, \mathbf{A}_{\mathrm{y}}\right)\right]_{:, \ell} ;\left[N_{\mathrm{y}}, N_{\mathrm{x}}\right]\right)^{T}$. We suppose that the $j$ th column of $\mathbf{P}^{(\ell)}$, i.e., $\mathbf{p}_{j}^{(\ell)} \in \mathbb{C}^{N_{\mathrm{x}}}$, contains $N_{j, \ell}^{\mathrm{c}}<N_{\mathrm{x}}$ indeterminate entries, indexed by $\left\{u_{1}, \ldots, u_{N_{j, \ell}^{\mathrm{c}}}\right\}$. Let $\overline{\mathbf{p}}_{j}^{(\ell)} \in \mathbb{C}^{N_{\mathrm{x}}}$ denote the column vector with indeterminate entries of $\mathbf{p}_{j}^{(\ell)}$ being replaced by zeros. Then, the columns of

$$
\mathbf{P}_{j}^{(\ell)}=\left[\overline{\mathbf{p}}_{j}^{(\ell)} /\left\|\overline{\mathbf{p}}_{j}^{(\ell)}\right\|, \mathbf{e}_{u_{1}}^{\left(N_{\mathrm{x}}\right)}, \ldots, \mathbf{e}_{u_{N_{j, \ell}^{\mathrm{c}}}^{\left(N_{\mathrm{x}}\right)}}^{(\ell)}\right] \in \mathbb{C}^{N_{\mathrm{x}} \times\left(1+N_{j, \ell}^{\mathrm{c}}\right)},
$$

constitute a basis of $\operatorname{range}\left(\mathbf{P}_{j}^{(\ell)}\right)$. By denoting the basis of $\operatorname{ker}\left(\mathbf{P}_{j}^{(\ell) H}\right)$ by $\mathbf{Q}_{j}^{(\ell)} \in \mathbb{C}^{N_{\mathrm{x}} \times\left(N_{\mathrm{x}}-1-N_{j, \ell}^{\mathrm{c}}\right)}$, the following relationship holds [41]

$$
\begin{aligned}
& \mathbf{a}_{\mathrm{x}, \ell} \in \bigcap_{j \in \mathcal{I}\left(N_{\mathrm{y}}\right)} \operatorname{range}\left(\mathbf{P}_{j}^{(\ell)}\right) \\
& \Leftrightarrow \mathbf{Q}_{j}^{(\ell) H} \mathbf{a}_{\mathrm{x}, \ell}=\mathbf{0}_{\left(N_{\mathrm{x}}-1-N_{j, \ell}^{\mathrm{c}}\right) \times 1}, \forall j \in \mathcal{I}\left(N_{\mathrm{y}}\right) .
\end{aligned}
$$

Hence, $\mathbf{A}_{\mathrm{x}}$ can be column-wisely derived as

$$
\mathbf{a}_{\mathbf{x}, \ell}^{\star}=\arg \min _{\left\|\mathbf{a}_{\mathbf{x}, \ell}\right\|_{2}^{2}=N_{\mathrm{x}}}\left\|\mathbf{a}_{\mathbf{x}, \ell}^{H}\left[\mathbf{Q}_{1}^{(\ell)}, \ldots, \mathbf{Q}_{N_{\mathrm{y}}}^{(\ell)}\right]\right\|_{2}^{2}, \forall \ell \in \mathcal{I}(L) \text {. }
$$

Since $\operatorname{dim}\left(\operatorname{ker}\left(\left[\mathbf{Q}_{1}^{(\ell)}, \ldots, \mathbf{Q}_{N_{\mathrm{y}}}^{(\ell)}\right]^{H}\right)\right)=1$, one can uniquely recover $\mathbf{a}_{\mathrm{x}, \ell}$ by solving (23). Now, we suppose that the $N_{j, \ell} \triangleq N_{\mathrm{x}}-N_{j, \ell}^{\mathrm{c}}$ determinate entries of $\mathbf{p}_{j}^{(\ell)}$ are indexed by $\left\{v_{1}, \ldots, v_{N_{j, \ell}}\right\}=\mathcal{I}\left(N_{\mathrm{x}}\right) \backslash\left\{u_{1}, \ldots, u_{N_{j, \ell}^{\mathrm{c}}}\right\}$. Define $\overline{\mathbf{a}}_{\mathrm{x}, \ell} \triangleq$ $\left[\mathbf{a}_{\mathrm{x}, \ell}\right]_{\left\{v_{1}, \ldots, v_{N_{j}, \ell}\right\}} \in \mathbb{C}^{N_{j, \ell}}$ and $\overline{\mathbf{p}}_{j}^{(\ell)} \triangleq\left[\mathbf{p}_{j}^{(\ell)}\right]_{\left\{v_{1}, \ldots, v_{N_{j, \ell}}\right\}} \in$ $\mathbb{C}^{N_{j, \ell}}$ as the observed parts of $\mathbf{a}_{\mathrm{x}, \ell}$ and $\mathbf{p}_{j}^{(\ell)}$ respectively. Then, the nonzero entries of $\mathbf{a}_{\mathrm{y}, \ell}$ can be computed as

$$
\left[\mathbf{a}_{\mathrm{y}, \ell}\right]_{j}=\overline{\mathbf{a}}_{\mathrm{x}, \ell}^{\dagger} \overline{\mathbf{p}}_{j}^{(\ell)}=\frac{\overline{\mathbf{a}}_{\mathrm{x}, \ell}^{H} \overline{\mathbf{p}}_{j}^{(\ell)}}{\left\|\overline{\mathbf{a}}_{\mathrm{x}, \ell}\right\|_{2}^{2}}, \forall j \in \mathcal{I}\left(N_{\mathrm{y}}\right) .
$$

After obtaining the estimation of the CPD factor matrices $\widehat{\mathbf{A}}_{\mathrm{x}}, \widehat{\mathbf{A}}_{\mathrm{y}}, \widehat{\mathbf{G}}_{\beta}$, the complete signal $\mathbf{Y}$, or equivalently the MSto-IRS narrowband channel $\left[\mathbf{h}_{1}, \ldots, \mathbf{h}_{P}\right]$, can be estimated as 
TABLE I

Statistical Mean of $\left\|\mathbf{d}_{\text {sel }}\right\|_{0}$ Vs. Number of Active Sensors

\begin{tabular}{|c|c|cccccccccc|}
\hline \multirow{2}{*}{$\mathbb{E}\left[\left\|\mathbf{d}_{\text {sel }}\right\|_{0}\right]$} & \multicolumn{10}{|c|}{$N_{\mathrm{A}}-\left(N_{\mathrm{x}}+N_{\mathrm{y}}-1\right)$} \\
\cline { 2 - 14 } & 2 & 4 & 6 & 8 & 10 & 12 & 14 & 16 & 18 & 20 \\
\hline \multirow{3}{*}{$N_{\mathrm{x}}, N_{\mathrm{y}}$} & 16 & 3.4 & 7.9 & 13.5 & 20.3 & 28.2 & 37.5 & 47.9 & 59.6 & 72.7 & 87.3 \\
& 24 & 3.2 & 7.3 & 12.0 & 17.5 & 23.8 & 30.8 & 38.6 & 47.3 & 56.6 & 66.7 \\
& 32 & 3.2 & 6.9 & 11.3 & 16.1 & 21.6 & 27.6 & 34.2 & 41.3 & 49.0 & 57.5 \\
\hline
\end{tabular}

$\left(\widehat{\mathbf{A}}_{\mathrm{x}} \odot \widehat{\mathbf{A}}_{\mathrm{y}}\right) \widehat{\mathbf{G}}_{\beta}^{T}$. Furthermore, the multipath AoAs $\left\{\phi_{\ell}, \theta_{\ell}\right\}_{\ell=1}^{L}$ can be estimated by leveraging the rotational-invariance feature of $\widehat{\mathbf{A}}_{\mathrm{x}}, \widehat{\mathbf{A}}_{\mathrm{y}}$ as

$$
\begin{gathered}
\hat{\omega}_{\mathrm{x}, \ell} \triangleq \sin \hat{\phi}_{\ell} \cos \hat{\theta}_{\ell}=\frac{\lambda}{2 \pi d} \measuredangle\left(\left[\hat{\mathbf{a}}_{\mathrm{x}, \ell}\right]_{1: N_{\mathrm{x}}-1}^{\dagger}\left[\hat{\mathbf{a}}_{\mathrm{x}, \ell}\right]_{2: N_{\mathrm{x}}}\right), \\
\hat{\omega}_{\mathrm{y}, \ell} \triangleq \sin \hat{\phi}_{\ell} \sin \hat{\theta}_{\ell}=\frac{\lambda}{2 \pi d} \measuredangle\left(\left[\hat{\mathbf{a}}_{\mathrm{y}, \ell}\right]_{1: N_{\mathrm{y}}-1}^{\dagger}\left[\hat{\mathbf{a}}_{\mathrm{y}, \ell}\right]_{2: N_{\mathrm{y}}}\right), \\
\hat{\theta}_{\ell}=\tan ^{-1} \frac{\hat{\omega}_{\mathrm{y}, \ell}}{\hat{\omega}_{\mathrm{x}, \ell}}, \hat{\phi}_{\ell}=\sin ^{-1} \frac{\hat{\omega}_{\mathrm{x}, \ell}}{\cos \hat{\theta}_{\ell}}=\sin ^{-1} \frac{\hat{\omega}_{\mathrm{y}, \ell}}{\sin \hat{\theta}_{\ell}},
\end{gathered}
$$

where $\measuredangle(\cdot)$ denotes the phase angle extraction operator. Finally, one can determine the scaling ambiguity of factor matrices and estimate the complex gains $\hat{\boldsymbol{\beta}}_{\ell} \triangleq\left[\hat{\beta}_{\ell, 1}, \ldots, \hat{\beta}_{\ell, P}\right]^{T}$ as $^{9}$

$$
\begin{aligned}
\hat{\boldsymbol{\beta}}_{\ell} & =\operatorname{Diag}(\mathbf{x})^{-1} \hat{\mathbf{g}}_{\beta, \ell}\left(\mathbf{a}_{\mathrm{x}}^{\dagger}\left(\hat{\phi}_{\ell}, \hat{\theta}_{\ell}\right) \hat{\mathbf{a}}_{\mathrm{x}, \ell}\right)\left(\mathbf{a}_{\mathrm{y}}^{\dagger}\left(\hat{\phi}_{\ell}, \hat{\theta}_{\ell}\right) \hat{\mathbf{a}}_{\mathrm{y}, \ell}\right) \\
& =\operatorname{Diag}(\mathbf{x})^{-1} \hat{\mathbf{g}}_{\beta, \ell}\left[\boldsymbol{\Lambda}_{\mathrm{x}}\right]_{\ell, \ell}\left[\boldsymbol{\Lambda}_{\mathrm{y}}\right]_{\ell, \ell}, \forall \ell \in \mathcal{I}(L),
\end{aligned}
$$

where $\mathbf{x} \triangleq\left[x_{1}, \ldots, x_{P}\right]^{T}$ concatenates the pilot symbols; $\left\{\boldsymbol{\Lambda}_{\mathrm{x}}, \boldsymbol{\Lambda}_{\mathrm{y}}\right\} \in \mathbb{C}^{L \times L}$ are the diagonal scaling ambiguities. Note that for the downlink case, we particularly design a set of precoded pilots as $\left\{\mathbf{F}_{q} \mathbf{x}_{q} \triangleq Q_{\mathrm{B}}^{-\frac{1}{2}} \mathbf{e}_{q}^{\left(N_{\mathrm{B}}\right)}\right\}_{q=1}^{Q_{\mathrm{B}}}$ with at least $M_{\mathrm{B}} \geq Q_{\mathrm{B}}+1$ RFCs [45]. Then, the $\ell$ th column of $\widehat{\mathbf{G}}_{\alpha} \in \mathbb{C}^{Q_{\mathrm{B}}} \bar{P} \times L_{0}$ equals $\mathbf{g}_{\alpha, \ell}=\left[\mathbf{a}_{\mathrm{B}}\left(\phi_{\mathrm{B}, \ell}, \theta_{\mathrm{B}, \ell}\right)\right]_{1: Q_{\mathrm{B}}} \otimes \boldsymbol{\alpha}_{\ell}$ with $\boldsymbol{\alpha}_{\ell} \triangleq\left[\alpha_{\ell, 1}, \ldots, \alpha_{\ell, P}\right]^{T}$. One can jointly estimate the entries of $\mathbf{a}_{\mathrm{B}}\left(\hat{\phi}_{\mathrm{B}, \ell}, \hat{\theta}_{\mathrm{B}, \ell}\right)$ and the complex gains $\hat{\boldsymbol{\alpha}}_{\ell}$ as

$$
\begin{aligned}
{\left[\mathbf{a}_{\mathrm{B}}\left(\hat{\phi}_{\mathrm{B}, \ell}, \hat{\theta}_{\mathrm{B}, \ell}\right)\right]_{q+1} } & =\left[\hat{\mathbf{g}}_{\alpha, \ell}\right]_{(q-1) P+1: q P}^{\dagger}\left[\hat{\mathbf{g}}_{\alpha, \ell}\right]_{q P+1:(q+1) P}, \\
\hat{\boldsymbol{\alpha}}_{\ell} & =\left[\hat{\mathbf{g}}_{\alpha, \ell}\right]_{1: P}, \forall q \in \mathcal{I}\left(Q_{\mathrm{B}}-1\right), \ell \in \mathcal{I}\left(L_{0}\right),
\end{aligned}
$$

where $\left\{\hat{\phi}_{\mathrm{B}, \ell}, \hat{\theta}_{\mathrm{B}, \ell}\right\}_{\ell=1}^{L_{0}}$ can be recovered according to the specific structure of BS antenna array.

We summarize the Fiber Sampling Tensor Completionbased channel estimation scheme as Algorithm 1, abbreviated as FS-TC. The algorithm only harnesses standard linear algebra and avoids iterative runs and random initialization, and equally importantly, it guarantees to return the exact solution in the noiseless case.

Remark. In practice, the calculations of kernel subspaces included in (19), (23) will suffer from the environment noise $\mathcal{N}$ in (7). Hence, in Step 4, we alternatively take the singular vectors corresponding to the smallest singular values of $\mathbf{S}_{\text {sel }} \mathbf{Q}_{2}(\overline{\mathcal{Z}}) \in \mathbb{C}^{\left\|\mathbf{d}_{\text {sel }}\right\|_{0} \times C_{L+1}^{2}}$ as its kernel subspace basis. Similarly, in Step 7, we take the left singular vector corresponding to the dominant singular value of $\left[\mathbf{P}_{1}^{(\ell)}, \ldots, \mathbf{P}_{N_{\mathrm{y}}}^{(\ell)}\right] \in$ $\mathbb{C}^{N_{\mathrm{x}} \times\left(N_{\mathrm{y}}+N_{\mathrm{P}}\right)}$ as the estimation of $\mathbf{a}_{\mathrm{x}, \ell}$. Note that $\mathbf{S}_{\mathrm{sel}} \mathbf{Q}_{2}(\overline{\mathcal{Z}})$

\footnotetext{
${ }^{9}$ In this procedure, the factors $\left\{\mathbf{a}_{\mathbf{x}, \ell}, \mathbf{a}_{\mathbf{y}, \ell}, \mathbf{g}_{\beta, \ell}\right\}_{\ell=1}^{L}$ are automatically paired with each other. One can ignore the column permutation ambiguity since it is identical for all the factor matrices.
}

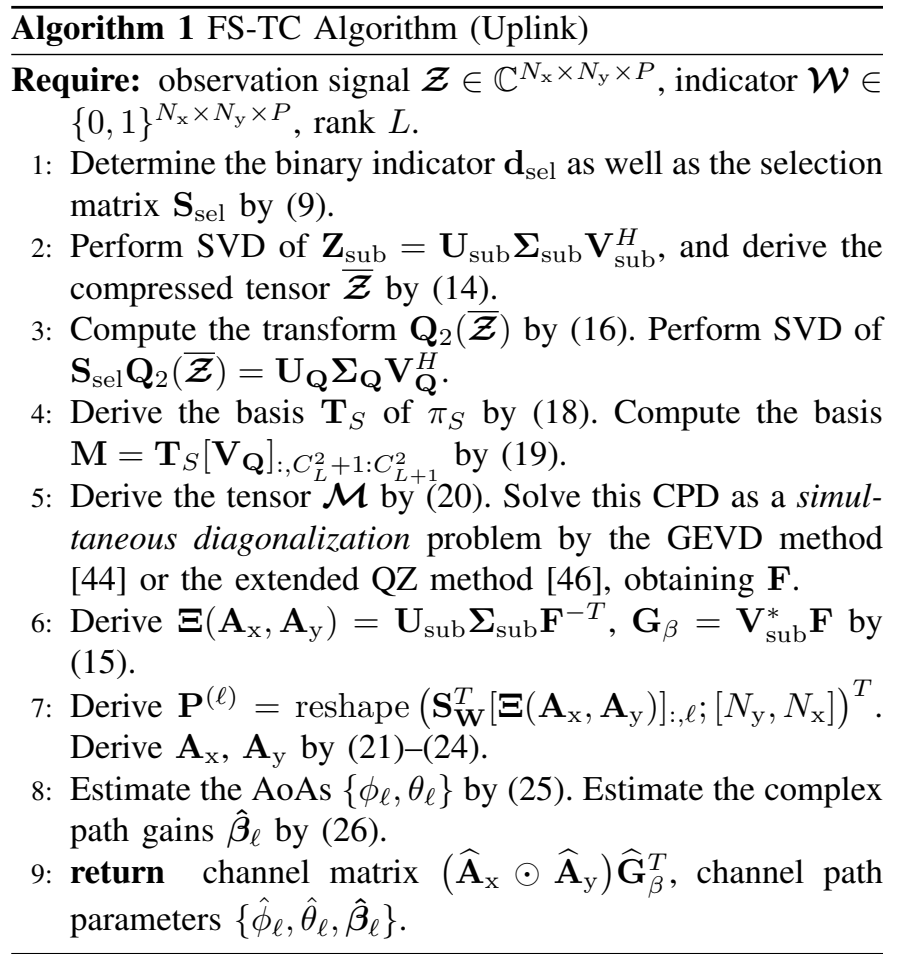

has a rank of $C_{L}^{2}$, while the SVD algorithm returns up to $\min \left(\left\|\mathbf{d}_{\text {sel }}\right\|_{0}, C_{L+1}^{2}\right)$ singular values and the corresponding singular vectors. In order to approximate the $L$-dimensional kernel subspace by $\left[\mathbf{V}_{\mathbf{Q}}\right]_{:, C_{L}^{2}+1: C_{L+1}^{2}}$ in step 4 , the condition $\left\|\mathbf{d}_{\text {sel }}\right\|_{0} \geq C_{L+1}^{2}$ requires to be satisfied, which is stricter than the uniqueness condition $\left\|\mathbf{d}_{\text {sel }}\right\|_{0} \geq C_{L}^{2}$ in (11). Considering the worst configuration as illustrated in Fig. 3(b), we suggest to activate $N_{\mathrm{A}} \geq N_{\mathrm{x}}+N_{\mathrm{y}}+C_{L+1}^{2}-1$ sensors for practical training to guarantee the validness of algorithms.

\section{Tensor Completion-Based Wideband Channel ESTIMATION}

In this section, we generalize the narrowband channel estimation approach to solve the wideband OFDM channel estimation problem, in which we can leverage the structural information of training signals in the frequency domain to relax the uniqueness conditions of tensor completion and develop the corresponding algorithms.

\section{A. Space-Time-Frequency Signal Tensor}

We represent the concatenated received incomplete uplink signal in (5) across $K$ subcarriers in $P$ time slots $\mathbf{Z}=$ 
$\left[\mathbf{z}_{1,1}, \ldots, \mathbf{z}_{P, 1}, \mathbf{z}_{1,2}, \ldots, \mathbf{z}_{P, K}\right] \in \mathbb{C}^{N_{\mathrm{I}} \times K P}$ as $^{10}$

$\mathbf{Z}=\mathbf{W} * \mathbf{Y}=\mathbf{W} *\left(\left(\mathbf{A}_{\mathrm{x}} \odot \mathbf{A}_{\mathrm{y}}\right)\left(\mathbf{P}_{\tau} \odot \mathbf{G}_{\beta}\right)^{T}\right)+\mathbf{W} * \mathbf{N}$

where $\mathbf{Y}(\mathbf{N}) \in \mathbb{C}^{N_{\mathrm{I}} \times K P}, \mathbf{W} \in\{0,1\}^{N_{\mathrm{I}} \times K P}$ denote the training signal (noise) and indicator, respectively; $\mathbf{P}_{\tau}=$ $\left[\mathbf{p}_{\tau, 1}, \ldots, \mathbf{p}_{\tau, L}\right] \in \mathbb{C}^{K \times L}$ and $\mathbf{G}_{\beta}=\left[\mathbf{g}_{\beta, 1}, \ldots, \mathbf{g}_{\beta, L}\right] \in$ $\mathbb{C}^{P \times L}$ with $\mathbf{p}_{\tau, \ell} \triangleq\left[1, \ldots, e^{-j 2 \pi \frac{(K-1) f_{\mathrm{s}} \tau_{\ell}}{K_{\mathrm{s}}}}\right]^{T}$ and $\mathbf{g}_{\beta, \ell} \triangleq$ $\left[\beta_{\ell, 1}, \ldots, \beta_{\ell, P}\right]^{T}$. According to $(41), \mathbf{Y}(\mathbf{Z})$ can be regarded as a tensor matricization, i.e., $\operatorname{Matr}(\mathcal{Y}(\mathcal{Z}) ;[2,1],[4,3])$, where

$$
\begin{aligned}
\mathcal{Y} & =\sum_{\ell=1}^{L} \mathbf{a}_{\mathrm{x}, \ell} \circ \mathbf{a}_{\mathrm{y}, \ell} \circ \mathbf{p}_{\tau, \ell} \circ \mathbf{g}_{\beta, \ell}+\mathcal{N} \\
& =\llbracket \mathbf{A}_{\mathrm{x}}, \mathbf{A}_{\mathrm{y}}, \mathbf{P}_{\tau}, \mathbf{G}_{\beta} \rrbracket+\mathcal{N}, \\
\mathcal{Z} & =\mathcal{W} * \mathcal{Y} \\
& =\mathcal{W} *\left(\sum_{\ell=1}^{L} \mathbf{a}_{\mathrm{x}, \ell} \circ \mathbf{a}_{\mathrm{y}, \ell} \circ \mathbf{p}_{\tau, \ell} \circ \mathbf{g}_{\beta, \ell}\right)+\mathcal{W} * \mathcal{N},
\end{aligned}
$$

with $\mathcal{W} \in\{0,1\}^{N_{\mathrm{x}} \times N_{\mathrm{y}} \times K \times P}$ and $\mathcal{N} \in\{0,1\}^{N_{\mathrm{x}} \times N_{\mathrm{y}} \times K \times P}$ being the tensorial forms of $\mathbf{W}$ and $\mathbf{N}$, respectively. By applying the row-selection matrix $\mathbf{S}_{\mathbf{W}} \in\{0,1\}^{N_{\mathrm{A}} \times N_{\mathrm{I}}}$ in (8), the effective observed signal submatrix $\mathbf{Z}_{\text {sub }} \in \mathbb{C}^{N_{\mathrm{A}} \times K P}$ can be similarly obtained as

$$
\mathbf{Z}_{\mathrm{sub}}=\mathbf{S}_{\mathbf{W}} \mathbf{Y}=\boldsymbol{\Xi}\left(\mathbf{A}_{\mathbf{x}} \odot \mathbf{A}_{\mathrm{y}}\right)\left(\mathbf{P}_{\tau} \odot \mathbf{G}_{\beta}\right)^{T}+\mathbf{S}_{\mathbf{W}} \mathbf{N} .
$$

One can verify that the $(i, j)$ th passive unit cell corresponds to an all-zero mode- $(3,4)$ slice $\mathbf{W}_{i, j,: \text { : }}$ as well as an all-zero

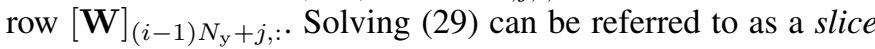
sampling CPD tensor completion problem.

\section{B. Uniqueness Condition of Tensor Completion}

We define the incomplete matrix $\mathbf{P}^{(\ell)}$ following (10). Then, by leveraging the structural characteristic of $\mathbf{P}_{\tau}$ in (28), we develop the following uniqueness condition of the fourth-order CPD tensor completion in (29).

Theorem 2. Consider a tensor $\mathcal{Y} \in \mathbb{C}^{N_{\mathrm{x}} \times N_{\mathrm{y}} \times K \times P}$ with factor matrices $\left\{\mathbf{A}_{\mathrm{x}}, \mathbf{A}_{\mathrm{y}}, \mathbf{P}_{\tau}, \mathbf{G}_{\beta}\right\}$, partially observed by $\mathcal{Z}$ in (29). Let $\widetilde{\mathbb{P}}^{(\ell)}$ be the restricted bipartite graph of $\mathbf{P}^{(\ell)}$ as defined in (10). If

$$
\left\{\begin{array}{l}
\operatorname{rank}\left(\boldsymbol{\Xi}\left(\mathbf{A}_{\mathrm{x}}, \mathbf{A}_{\mathrm{y}}\right)\right)=\operatorname{rank}\left(\mathbf{P}_{\tau}^{(K-1)} \odot \mathbf{G}_{\beta}\right)=L, \\
\tau_{\ell_{1}} \neq \tau_{\ell_{2}}, \forall \ell_{1} \neq \ell_{2} \in \mathcal{I}(L), \\
\widetilde{\mathbb{P}}^{(\ell)} \text { is a connected graph, } \forall \ell \in \mathcal{I}(L),
\end{array}\right.
$$

where $\mathbf{P}_{\tau}^{(K-1)} \triangleq\left[\mathbf{P}_{\tau}\right]_{1: K-1,:}$, then the rank of $\mathcal{Y}$ equals $L$, and its CPD is unique up to scaling and permutation ambiguity.

Proof: See Appendix B.

In different cellular communication scenarios, some signal propagation paths with different AoAs coincidentally experience identical time delay. In this case, Theorem 2 is no

\footnotetext{
${ }^{10}$ One default configuration is to insert identical pilots, e.g, $x_{p, k}=1$, into consecutive subcarriers. Actually, all the common comb-type pilot arrangements are permitted, which only cause a minor variation of the algorithm. Moreover, one may use rotational-invariant pilots, e.g., $\left\{x_{p, k} \triangleq\right.$ $\left.e^{(k-1) \delta}\right\}_{k=1}^{K}$, to better control the peak-to-average power ratio.
}

longer applicable. Fortunately, we can leverage the concept of virtual space-frequency array to develop another uniqueness condition. Define

$$
\begin{aligned}
& \widetilde{\mathbf{A}}_{\mathrm{x}} \triangleq \mathbf{P}_{\tau}^{\left(K_{\mathrm{x}}\right)} \odot \mathbf{A}_{\mathrm{x}}, \tilde{\mathbf{a}}_{\mathrm{x}, \ell}=\mathbf{p}_{\tau, \ell}^{\left(K_{\mathrm{x}}\right)} \otimes \mathbf{a}_{\mathrm{x}, \ell}, \\
& \widetilde{\mathbf{A}}_{\mathrm{y}} \triangleq \mathbf{P}_{\tau}^{\left(K_{\mathrm{y}}\right)} \odot \mathbf{A}_{\mathrm{y}}, \tilde{\mathbf{a}}_{\mathrm{y}, \ell}=\mathbf{p}_{\tau, \ell}^{\left(K_{\mathrm{y}}\right)} \otimes \mathbf{a}_{\mathrm{y}, \ell},
\end{aligned}
$$

where $K_{\mathrm{x}}+K_{\mathrm{y}}=K+1, \mathbf{p}_{\tau, \ell}^{\left(K_{\mathrm{x}}\right)}=\left[\mathbf{P}_{\tau}^{\left(K_{\mathrm{x}}\right)}\right]_{:, \ell}, \mathbf{p}_{\tau, \ell}^{\left(K_{\mathrm{y}}\right)}=$ $\left[\mathbf{P}_{\tau}^{\left(K_{\mathrm{y}}\right)}\right]_{:, \ell}$. Then, we determine the binary indicator $\tilde{\mathbf{d}}_{\text {sel }} \in$ $\{0,1\}^{C_{K_{\mathrm{x}} N_{\mathrm{x}}}^{2} C_{K_{\mathrm{y}} N_{\mathrm{y}}}^{2} \text { as }}$

$$
\begin{aligned}
\tilde{\mathbf{d}}_{\mathrm{sel}}= & {\left[\tilde{d}_{(1,2),(1,2)}, \tilde{d}_{(1,2),(1,3)}, \ldots,\right.} \\
& \left.\tilde{d}_{\left(K_{\mathrm{x}} N_{\mathrm{x}}-1, K_{\mathrm{x}} N_{\mathrm{x}}\right),\left(K_{\mathrm{y}} N_{\mathrm{y}}-1, K_{\mathrm{y}} N_{\mathrm{y}}\right)}\right]^{T}, \\
\tilde{d}_{\left(i_{1}, i_{2}\right),\left(j_{1}, j_{2}\right)}= & \left\{\begin{array}{c}
1, \text { if } \tilde{\mathbf{y}}_{i_{1}, j_{1},:}, \tilde{\mathbf{y}}_{i_{1}, j_{2},:}, \tilde{\mathbf{y}}_{i_{2}, j_{1},:}, \tilde{\mathbf{y}}_{i_{2}, j_{2},:} \\
\text { are observable fibers, } \\
0, \text { otherwise, }
\end{array}\right.
\end{aligned}
$$

where the fibers $\left\{\tilde{\mathbf{y}}_{i, j,:}\right\} \in \mathbb{C}^{P}$ are from $\widetilde{\mathcal{Y}} \triangleq \llbracket \widetilde{\mathbf{A}}_{\mathrm{x}}, \widetilde{\mathbf{A}}_{\mathrm{y}}, \mathbf{G}_{\beta} \rrbracket \in$ $\mathbb{C}^{K_{\mathrm{x}} N_{\mathrm{x}} \times K_{\mathrm{y}} N_{\mathrm{y}} \times P}$. Now, another new uniqueness condition of CPD tensor completion is presented as follows:

Theorem 3. Consider a tensor $\mathcal{Y} \in \mathbb{C}^{N_{\mathrm{x}} \times N_{\mathrm{y}} \times K \times P}$ with factor matrices $\left\{\mathbf{A}_{\mathrm{x}}, \mathbf{A}_{\mathrm{y}}, \mathbf{P}_{\tau}, \mathbf{G}_{\beta}\right\}$, partially observed by $\mathcal{Z}$ in (29). Let $\widetilde{\mathbf{D}}_{\text {sel }}=\operatorname{Diag}\left(\tilde{\mathbf{d}}_{\text {sel }}\right)$ as defined in (33), and let $\widetilde{\mathbb{P}}^{(\ell)}$ be the restricted bipartite graph of $\mathbf{P}^{(\ell)}$ as defined in (10). If

$$
\left\{\begin{array}{l}
\operatorname{rank}\left(\mathbf{G}_{\beta}\right)=L, \\
\operatorname{rank}\left(\mathbf{D}_{\text {sel }}\left(\mathbf{C}_{2}\left(\widetilde{\mathbf{A}}_{\mathrm{x}}\right) \odot \mathbf{C}_{2}\left(\widetilde{\mathbf{A}}_{\mathrm{y}}\right)\right)\right)=C_{L}^{2}, \\
\widetilde{\mathbb{P}}^{(\ell)} \text { is a connected graph, } \forall \ell \in \mathcal{I}(L),
\end{array}\right.
$$

with $\widetilde{\mathbf{A}}_{\mathrm{x}}, \widetilde{\mathbf{A}}_{\mathrm{y}}$ defined as in (32), then the rank of $\mathcal{Y}$ equals $L$, and its CPD is unique up to scaling and permutation ambiguities.

Proof: See Appendix C.

Remark. In the generic case, the first condition of (31) becomes $\min \left(N_{\mathrm{A}},(K-1) P\right) \geq L$. On the other hand, as illustrated in Fig. 4, each of the $N_{\mathrm{A}}$ determinate entries of $\mathbf{P}^{(\ell)}$ in (10) corresponds to a $K_{\mathrm{x}} \times K_{\mathrm{y}}$ effective submatrix in the virtual array planes $\tilde{\mathbf{a}}_{\mathrm{x}, \ell} \tilde{\mathbf{a}}_{\mathrm{y}, \ell}^{T}$, which significantly increases $\left\|\tilde{\mathbf{d}}_{\text {sel }}\right\|_{0}$ in (33). Therefore, Theorem 2 and 3 indicate that introducing structural information of training signals in the frequency domain, i.e., $\mathbf{P}_{\tau}$, helps relax the uniqueness condition of CPD tensor completion.

\section{Proposed Algorithms}

The Slice Sampling Tensor Completion-based wideband channel estimation scheme is summarized as Algorithm 2, abbreviated as SS-TC. This approach contains two schemes respectively derived from Theorem 2 and 3 .

Remark. If $K_{\mathrm{x}} \approx K_{\mathrm{y}}$, one can define $K_{\mathrm{m}} \triangleq \min \left(K_{\mathrm{x}}, K_{\mathrm{y}}\right)$ and compute

$$
\begin{aligned}
\widetilde{\mathbf{A}}_{\mathrm{xy}} \triangleq & {\left[\left[\widetilde{\mathbf{A}}_{\mathrm{x}}\right]_{1: N_{\mathrm{x}},:}^{T},\left[\widetilde{\mathbf{A}}_{\mathrm{y}}\right]_{1: N_{\mathrm{y}},:}^{T}, \ldots,\right.} \\
& {\left.\left[\widetilde{\mathbf{A}}_{\mathrm{x}}\right]_{\left(K_{\mathrm{m}}-1\right) N_{\mathrm{x}}+1: K_{\mathrm{m}} N_{\mathrm{x}},:}^{T},\left[\widetilde{\mathbf{A}}_{\mathrm{y}}\right]_{\left(K_{\mathrm{m}}-1\right) N_{\mathrm{y}}+1: K_{\mathrm{m}} N_{\mathrm{y}},:}^{T}\right]^{T} } \\
= & \mathbf{P}_{\tau}^{\left(K_{\mathrm{m}}\right)} \odot\left[\begin{array}{ll}
\mathbf{A}_{\mathrm{x}}^{T} & \mathbf{A}_{\mathrm{y}}^{T}
\end{array}\right]^{T},
\end{aligned}
$$




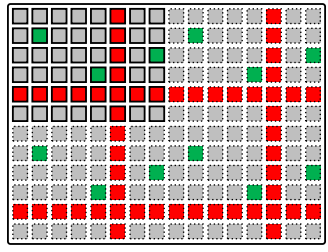

(a) Virtual array-type I

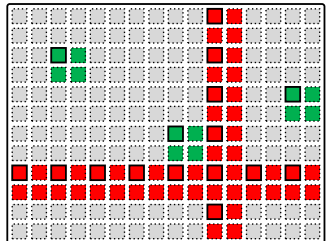

(b) Virtual array-type II
Fig. 4. Diagram of a virtual space-frequency array plane of hybrid IRS with $K_{\mathrm{x}}=K_{\mathrm{y}}=2$. The gray/red/green squares denote the passive/basic active/extra active entries; the solid/dotted boxes denote the physical/virtual IRS elements. (a) $\left\{\mathbf{p}_{\tau, \ell}^{\left(K_{\mathrm{x}}\right)} \otimes \mathbf{a}_{\mathrm{x}, \ell}\right\} \times\left\{\mathbf{p}_{\tau, \ell}^{\left(K_{\mathrm{y}}\right)} \otimes \mathbf{a}_{\mathrm{y}, \ell}\right\}$-plane. (b) Equivalent $\left\{\mathbf{a}_{\mathrm{x}, \ell} \otimes \mathbf{p}_{\tau, \ell}^{\left(K_{\mathrm{x}}\right)}\right\} \times\left\{\mathbf{a}_{\mathrm{y}, \ell} \otimes \mathbf{p}_{\tau, \ell}^{\left(K_{\mathrm{y}}\right)}\right\}$-plane.

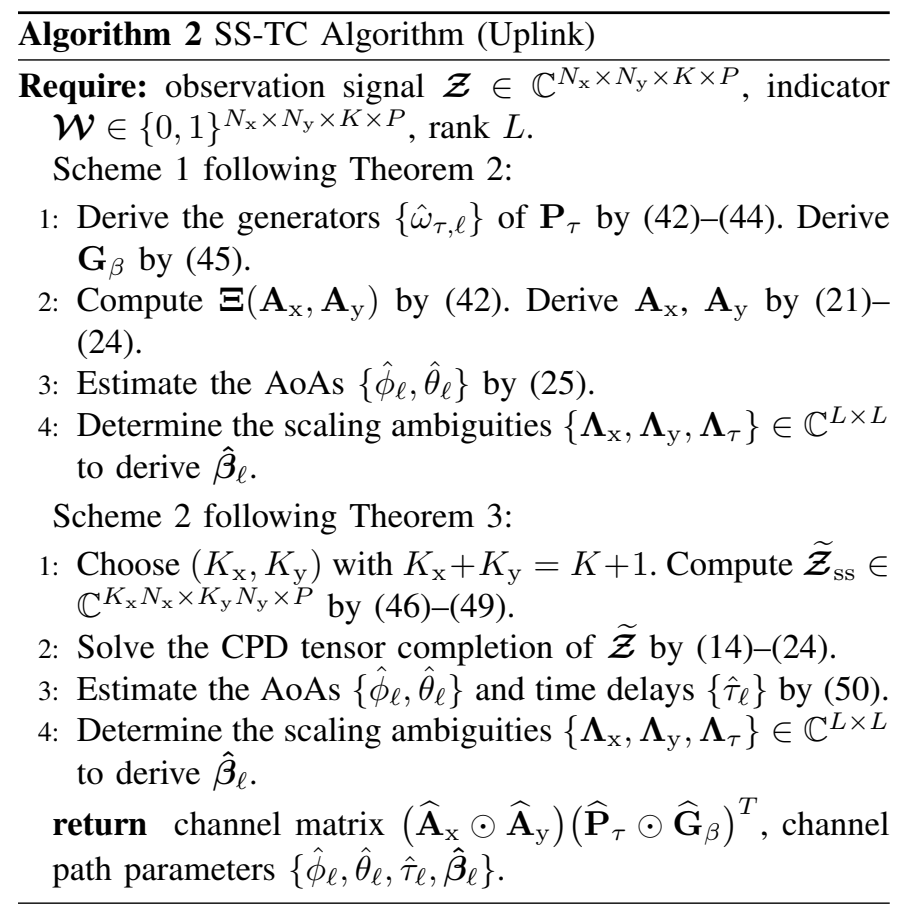

to estimate $\left\{\omega_{\tau, \ell}\right\}_{\ell=1}^{L}$ by leveraging the rotational-invariance property of (35).

Moreover, if the system adopts a common comb-type pilot arrangement that does not satisfy the rotational-invariance property, neither Theorem 2 nor 3 applies. In this case, we can directly solve the third-order CPD $\llbracket \mathbf{A}_{\mathrm{x}}, \mathbf{A}_{\mathrm{y}}, \mathbf{P}_{\tau} \odot \mathbf{G}_{\beta} \rrbracket$ of (29) according to Theorem 1, and solve a set of $L$ rank-1 factorization subproblems as

$$
\left\{\mathbf{p}_{\tau, \ell}^{\star}, \mathbf{g}_{\beta, \ell}^{\star}\right\}=\arg \max _{\left\|\mathbf{p}_{\tau, \ell}\right\|_{2}^{2}=K, \mathbf{g}_{\beta, \ell}}\left\|\boldsymbol{\Omega}^{(\ell)}-\mathbf{p}_{\tau, \ell} \mathbf{g}_{\beta, \ell}^{T}\right\|_{F}^{2},
$$

where $\boldsymbol{\Omega}^{(\ell)}=\operatorname{reshape}\left(\left[\mathbf{P}_{\tau} \odot \mathbf{G}_{\beta}\right]_{i, \ell} ;[P, K]\right)^{T} \in \mathbb{C}^{K \times P}$. One can take the dominant left/right singular vectors of $\boldsymbol{\Omega}^{(\ell)}$ as the solution of (36).

\section{Numerical Results}

\section{A. Computational Complexity}

In Algorithm 1, the complexity is dominated by the construction of $\mathbf{S}_{\mathrm{sel}} \mathbf{Q}_{2}(\overline{\mathcal{Z}})$ and the computation of the CPD $\mathcal{M}$, which are of the order $\mathcal{O}\left(\left\|\mathbf{d}_{\text {sel }}\right\|_{0} C_{L+1}^{2}\right)$ and $\mathcal{O}\left(L^{3}\right)$ respectively. In Algorithm 2, the complexity of Solution 1 is dominated by the derivation of $\mathbf{P}_{\tau}, \mathbf{G}_{\beta}$, which is of the order $\mathcal{O}\left(K P L^{2}\right)$; the complexity of Solution 2 is dominated by the computation of the CPD $\widetilde{\mathcal{Z}}$, which is of the order $\mathcal{O}\left(\left\|\tilde{\mathbf{d}}_{\text {sel }}\right\|_{0} C_{L+1}^{2}+L^{3}\right)$. As a comparison, the CS method [23] has a complexity of the order $\mathcal{O}\left(N_{\mathrm{x}} N_{\mathrm{y}} P\left(N_{\mathrm{x}}+N_{\mathrm{y}}\right)\right)$; the Alternating Least Squares (ALS) [28] has a complexity of the order $\mathcal{O}\left(3 L^{2} N_{\mathrm{A}}+L^{3}\left(N_{\mathrm{x}}+N_{\mathrm{y}}+P\right)\right)$ per iteration; the Tensor completion by parallel Matrix fACtorization (TMac) [29] has a complexity of the order $\mathcal{O}\left(L N_{\mathrm{x}} N_{\mathrm{y}} P+L^{2}\left(N_{\mathrm{x}}+N_{\mathrm{y}}+P\right)\right)$ per iteration; the optimization-based nonlinear least squares (NLS) [30] has a complexity of the order $\mathcal{O}\left(L^{2} N_{\mathrm{A}}+L^{3} N_{\mathrm{x}} N_{\mathrm{y}} P\right)$ per iteration.

\section{B. Simulation Results}

All the typical simulation parameters are listed here: $N_{\mathrm{x}}=$ $N_{\mathrm{y}}=16$ or $32 ; N_{\mathrm{B}}=32(\mathrm{ULA}), M_{\mathrm{B}}=3 ; Q_{\mathrm{M}}=1$, $Q_{\mathrm{B}}=2 ; K_{\mathrm{s}}=128, f_{\mathrm{c}}=28 \mathrm{GHz}, L_{0}=L=4, U=1$; the AoD/AoAs $\left\{\phi_{\mathrm{B}(\mathrm{I}, u), \ell}, \theta_{\mathrm{B}(\mathrm{I}, u), \ell}\right\}$ follow the uniform distribution $\mathcal{U}\left(-\frac{\pi}{2}, \frac{\pi}{2}\right)$; the complex gains $\left\{\alpha_{\ell, p}, \beta_{u, \ell, p}\right\}$ follow the normal distribution $\mathcal{C N}(0,1)$; the time delays $\left\{\tau_{0(u), \ell}\right\}$ follow the uniform distribution $\mathcal{U}\left(0,10^{2} \mathrm{~ns}\right)$. The proposed FS-TC and SS-TC schemes are compared with the CS [23], ALS [28], TMac [29] and NLS [30]. ${ }^{11}$

1) Uplink Narrowband Training: Table II tabulates the algorithm computational complexity quantified by the single running time (milliseconds) as well as the effective channel estimation performance evaluated by the normalized mean square error (NMSE). ${ }^{12}$ It shows that the proposed FS-TC can achieve enhanced performance with the lowest complexity, and the running time is inversely proportional to the number of missing signal fibers, or equivalently the missing ratio (MR) $N_{\mathrm{P}} / N_{\mathrm{I}}$. The performance and complexity of the iterative counterparts suffer from either the MR/noise level or the IRS scale, respectively. On the contrary, the FS-TC performs robustly against those hardware and system factors.

Fig. 5 plots the NMSE curves of channel matrices versus the received SNR, i.e., $\|\mathcal{Y}-\mathcal{N}\|_{F}^{2} /\|\mathcal{N}\|_{F}^{2}$. It indicates that the FS-TC outperforms the ALS/TMac and CS when the SNR increases to $15 \mathrm{~dB}$ and $22.5 \mathrm{~dB}$, respectively, while it can also achieve performance close to that of the NLS in the high-SNR region with much faster speed. Moreover, the performance of FS-TC is exponentially improved against the increasing SNR, while the CS and ALS cannot benefit much from the SNR. It can also be observed that even with a higher MR, the performance of the second configuration is better than that of the first one. This indicates that the number of effective signal measurements is more significant than the sampling ratio for the recovery of partially observed signal tensors.

\footnotetext{
${ }^{11}$ To better evaluate the estimation performance of channel parameters, the tensor rank $L_{0}, L$ is assumed to be known or perfectly estimated a priori for all the approaches. The angle resolution of CS method is $\frac{2 \pi}{8 N_{\mathrm{x}}} \times \frac{2 \pi}{8 N_{\mathrm{y}}}$.

${ }^{12}$ The NMSE metrics for narrowband and wideband uplink training are defined as $\left\|\left(\mathbf{A}_{\mathbf{x}} \odot \mathbf{A}_{\mathrm{y}}\right) \mathbf{G}_{\beta}^{T}-\left(\widehat{\mathbf{A}}_{\mathbf{x}} \odot \widehat{\mathbf{A}}_{\mathrm{y}}\right) \widehat{\mathbf{G}}_{\beta}^{T}\right\|_{F}^{2} /\left\|\left(\mathbf{A}_{\mathbf{x}} \odot \mathbf{A}_{\mathrm{y}}\right) \mathbf{G}_{\beta}^{T}\right\|_{F}^{2}$ and $\left\|\left(\mathbf{A}_{\mathbf{x}} \odot \mathbf{A}_{\mathbf{y}}\right)\left(\mathbf{P}_{\tau} \odot \mathbf{G}_{\beta}\right)^{T}-\left(\widehat{\mathbf{A}}_{\mathbf{x}} \odot \widehat{\mathbf{A}}_{\mathbf{y}}\right)\left(\widehat{\mathbf{P}}_{\tau} \odot \widehat{\mathbf{G}}_{\beta}\right)^{T}\right\|_{F}^{2} / \|\left(\mathbf{A}_{\mathbf{x}} \odot \mathbf{A}_{\mathbf{y}}\right)\left(\mathbf{P}_{\tau} \odot\right.$ $\left.\mathbf{G}_{\beta}\right)^{T} \|_{F}^{2}$, respectively. The NMSE of downlink training is similarly defined. The simulation laptop configuration is Intel(R) Core(TM) i7-8750H CPU $2.20 \mathrm{GHz}, 8.00 \mathrm{~GB}$ RAM; the notation "- " means that the algorithm cannot return effective estimates with NMSE $<1.0$.
} 
TABLE II

Performance Comparisons of Tensor Completion APPROACHES

\begin{tabular}{|c|c|c|c|c|c|c|c|c|c|c|c|}
\hline \multicolumn{2}{|c|}{ Approaches } & \multicolumn{2}{|c|}{ CS [23] } & \multicolumn{2}{|c|}{ ALS [28] } & \multicolumn{2}{|c|}{ TMac [29] } & \multicolumn{2}{|c|}{ NLS [30] } & \multicolumn{2}{|c|}{ FS-TC } \\
\hline $\begin{array}{l}\text { MR } \\
\text { (\%) }\end{array}$ & $\begin{array}{l}\text { SNR } \\
\text { (dB) }\end{array}$ & err & time & err & time & err & time & err & time & err & time \\
\hline \multicolumn{12}{|c|}{$\llbracket N_{\mathrm{x}}, N_{\mathrm{y}}, P \rrbracket_{L}=\llbracket 16,16,6 \rrbracket_{4}$} \\
\hline \multirow{2}{*}{75} & 25 & $1.1 \mathrm{e}-2$ & $3.0 \mathrm{e}+1$ & $4.6 \mathrm{e}-1$ & $4.2 \mathrm{e}+1$ & $1.3 \mathrm{e}-2$ & $2.3 \mathrm{e}+2$ & 4.8e-3 & $4.0 \mathrm{e}+3$ & $5.4 \mathrm{e}-3$ & $3.3 \mathrm{e}+1$ \\
\hline & 40 & $1.0 \mathrm{e}-2$ & $3.0 \mathrm{e}+1$ & $3.7 \mathrm{e}-1$ & $4.2 \mathrm{e}+1$ & $2.2 \mathrm{e}-3$ & $2.2 \mathrm{e}+2$ & $1.4 \mathrm{e}-4$ & $3.9 \mathrm{e}+3$ & $1.6 e-4$ & $3.2 \mathrm{e}+1$ \\
\hline \multirow{2}{*}{80} & 25 & $1.2 \mathrm{e}-2$ & $2.8 \mathrm{e}+1$ & - & $3.9 \mathrm{e}+1$ & $3.0 \mathrm{e}-1$ & $2.3 e+2$ & $6.4 \mathrm{e}-2$ & $4.7 e+3$ & 8.9e-3 & $2.6 e+1$ \\
\hline & 40 & $1.1 \mathrm{e}-2$ & $2.7 \mathrm{e}+1$ & - & $3.8 \mathrm{e}+1$ & $2.8 \mathrm{e}-1$ & $2.4 \mathrm{e}+2$ & $2.7 \mathrm{e}-3$ & $4.5 e+3$ & $2.5 \mathrm{e}-4$ & $2.5 e+1$ \\
\hline \multicolumn{12}{|c|}{$\llbracket N_{\mathrm{x}}, N_{\mathrm{y}}, P \rrbracket_{L}=\llbracket 32,32,6 \rrbracket_{4}$} \\
\hline \multirow{2}{*}{85} & 25 & $8.5 \mathrm{e}-3$ & $4.6 e+2$ & $3.3 \mathrm{e}-1$ & $2.9 \mathrm{e}+2$ & $6.5 \mathrm{e}-3$ & $5.3 \mathrm{e}+2$ & 3.1e-3 & $4.1 \mathrm{e}+3$ & $3.8 \mathrm{e}-3$ & $1.1 \mathrm{e}+2$ \\
\hline & 40 & $8.2 \mathrm{e}-3$ & $4.4 \mathrm{e}+2$ & $2.4 \mathrm{e}-1$ & $3.0 \mathrm{e}+2$ & $1.1 \mathrm{e}-3$ & $5.2 \mathrm{e}+2$ & $1.2 \mathrm{e}-4$ & $4.1 \mathrm{e}+3$ & $1.3 \mathrm{e}-4$ & $1.2 \mathrm{e}+2$ \\
\hline \multirow{2}{*}{90} & 25 & $9.3 e-3$ & $3.4 \mathrm{e}+2$ & - & $2.3 e+2$ & $5.4 \mathrm{e}-1$ & $5.0 \mathrm{e}+2$ & - & $5.5 e+3$ & 7.6e-3 & $8.6 e+1$ \\
\hline & 40 & $9.2 \mathrm{e}-3$ & $3.2 \mathrm{e}+2$ & - & $2.3 e+2$ & $4.6 \mathrm{e}-1$ & $5.1 \mathrm{e}+2$ & $9.2 \mathrm{e}-1$ & $5.4 e+3$ & $1.7 e-4$ & $8.7 \mathrm{e}+1$ \\
\hline
\end{tabular}

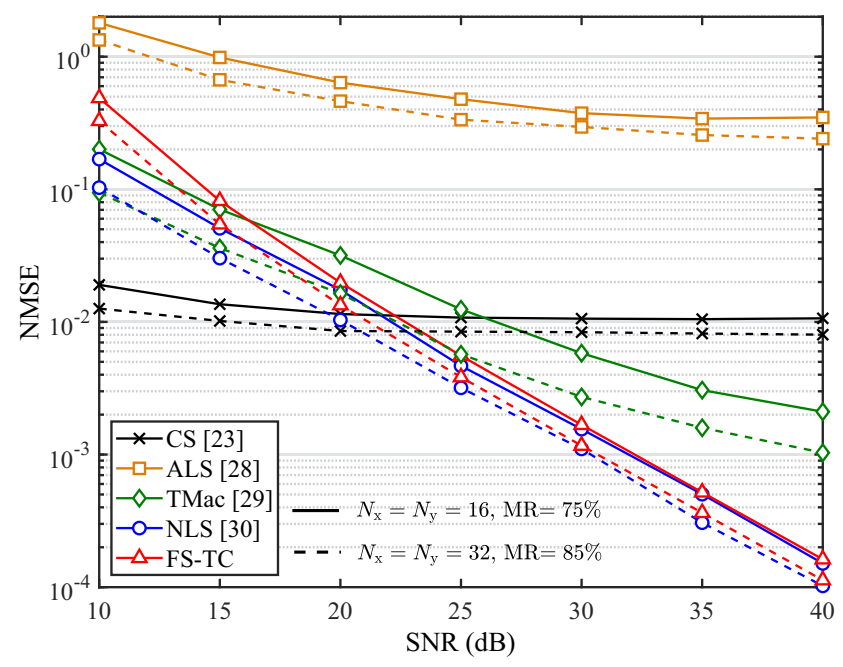

Fig. 5. Channel NMSE performance vs. SNR, $P=6$.

Fig. 6 plots the NMSE curves versus the MR of hybrid IRS. It illustrates that the performance of all the approaches, especially that of the ALS, TMac and NLS, deteriorates against the increasing MR. For the case where the number of effective signal samples is extremely limited, the FS-TC shows its superior performance. Concretely, when the MR exceeds $75 \%$ for the $16 \times 16$-layout and $85 \%$ for the $32 \times 32$-layout, the FS-TC evidently outperforms most of the counterparts. The robustness and short running time make FS-TC perfectly suitable for low-complexity IRSs with limited number of active elements, which can efficiently reduce the hardware complexity and power consumption.

Fig. 7 plots the NMSE curves versus the number of training frames. It shows that except the CS method, all the other methods can achieve enhanced estimation accuracy against the increasing $P$. This is due to the fact that more training time slots extend the depth of observation signal fibers to improve the performance of tensor completion approaches, which corroborates the analysis of uniqueness condition, while the performance of CS mainly depends on the resolution of sensing codebook. Furthermore, it can be observed that as $P$ increases, the rate of performance enhancement gradually decreases.

Fig. 8 plots the number of training frames versus the

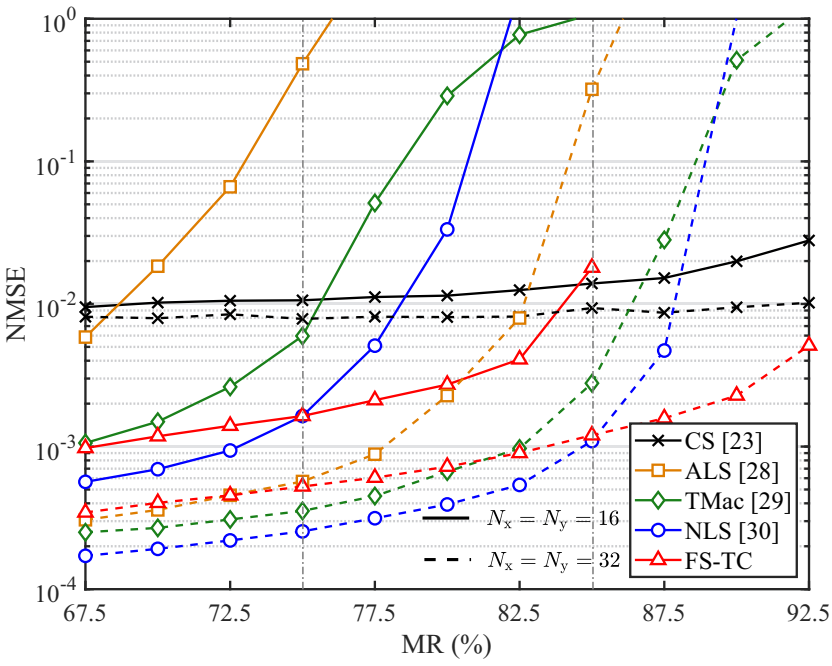

Fig. 6. Channel NMSE performance vs. MR, $P=6, \mathrm{SNR}=30 \mathrm{~dB}$.

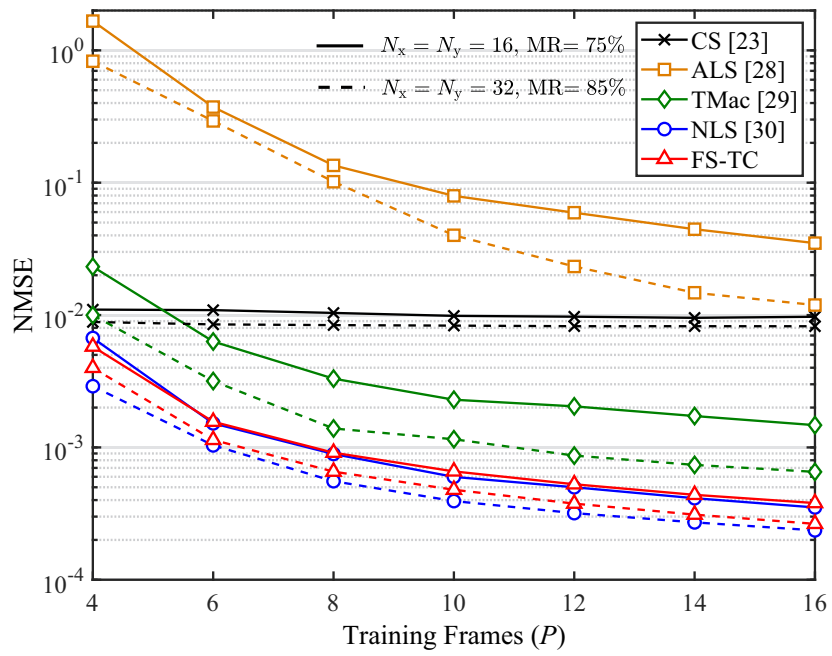

Fig. 7. Channel NMSE performance vs. training frames, $\mathrm{SNR}=30 \mathrm{~dB}$.

received SNR, given a target NMSE. It shows that the as the SNR gradually increases, the minimal number of measurements $P$ required to achieve desired performance decreases exponentially by orders of magnitude. Concretely, given an objective NMSE, the maximal SNR gap that can be compen- 


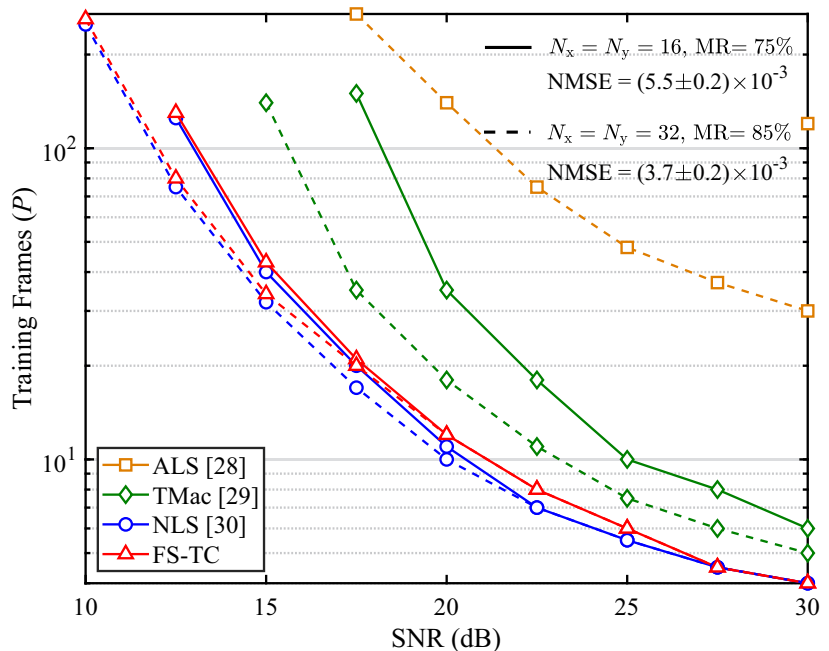

Fig. 8. Training frames vs. SNR given a target NMSE.

sated by adjusting the quantity of measurements is $10 \mathrm{~dB}, 15$ $\mathrm{dB}$ and $20 \mathrm{~dB}$ for ALS, TMac and NLS/FS-TC respectively, whilst this considerable gap narrows down as the number of effective samples $N_{\mathrm{A}}$ reduces.

2) Downlink Wideband Training: Fig. 9 plots the NMSE curves of downlink wideband training versus the SNR. ${ }^{13}$ It demonstrates that the SS-TC schemes achieve exponentially improved performance against the increasing SNR, and systematically outperform the other counterparts at the highSNR region. Moreover, as the bandwidth increases, the SSTC-1 returns significantly enhanced results, while the SSTC-2 is relatively insensitive to $f_{\mathrm{s}}$. This can be explained as follows: an increasing $f_{\mathrm{s}}$ (or decreasing $K_{\mathrm{s}}$ ) enlarges the subcarrier interval, as well as, the statistical phase distances of $\left\{e^{-j 2 \pi f_{\mathrm{s}} \tau_{0, \ell} / K_{\mathrm{s}}}\right\}$ to improve the resolution of $\left\{\tau_{0, \ell}, \alpha_{\ell, p}\right\}$ in the SS-TC-1, which directly determines the recovery accuracy of $\mathbf{A}_{\mathrm{x}(\mathrm{y})}$. In the SS-TC-2 method, the information of time delays has been integrated into the virtual space-frequency arrays and gets recovered after the AoAs, which contributes much less to channel matrix estimation.

Fig. 10 plots the rooted mean square error (RMSE) curves of multipath parameters, i.e., $\left\{\phi_{\mathrm{I}, \ell}, \theta_{\mathrm{I}, \ell}, \phi_{\mathrm{B}, \ell}, \tau_{0, \ell}\right\}$ versus the SNR. ${ }^{14}$ One can observe that the recovery accuracy achieved by the SS-TC methods exponentially improves against the increasing SNR. It also shows that the increasing bandwidth improves the RMSEs of all the parameters, especially the time delays, in the SS-TC-1, whilst those of AoA/AoDs in the SS-TC-2 remain relatively stable. This phenomenon conforms with the previous frequency analysis of Fig. 9. Combining with the complexity analysis, we can infer that the SS-TC-1 is suitable for large bandwidths with numerous training subcarriers, while the SS-TC-2 is oppositely suitable for the case with small values of $K$ and $f_{\mathrm{s}}$. By contrast, the CS method returns

\footnotetext{
${ }^{13}$ The scheme 1 and 2 in Algorithm 2 are denoted by SS-TC-1 and SS-TC2 , respectively. The ALS method does not effectively work, and therefore, its performance curve is omitted.

${ }^{14}$ The RMSE of parameter $x_{\ell}$ is defined as $\sqrt{\frac{1}{L_{0}} \sum_{\ell=1}^{L_{0}}\left(x_{\ell}-\hat{x}_{\ell}\right)^{2}}$. The CS method cannot factorize the frequency and time parameters, and the TMac method computes the whole tensor signal but cannot derive the factor matrices.
}

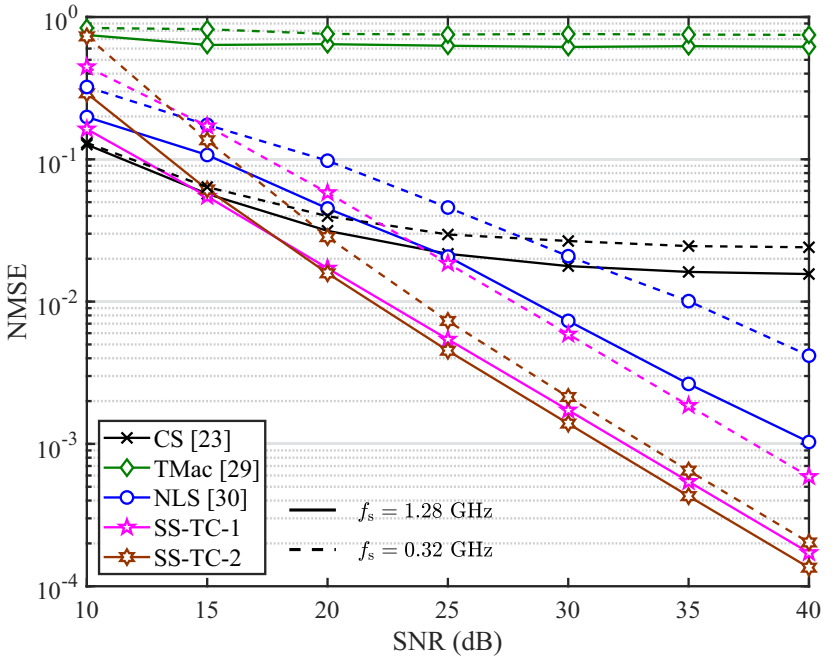

Fig. 9. Channel NMSE performance vs. SNR, $N_{\mathrm{x}}=N_{\mathrm{y}}=16, \mathrm{MR}=$ $82.5 \%, P=6, K=3$.

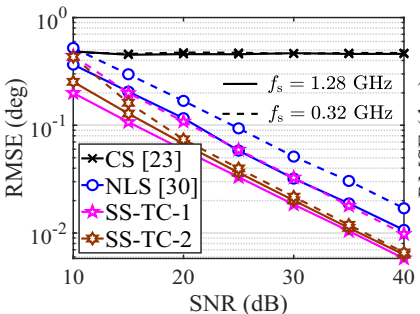

(a) Azimuth AoA

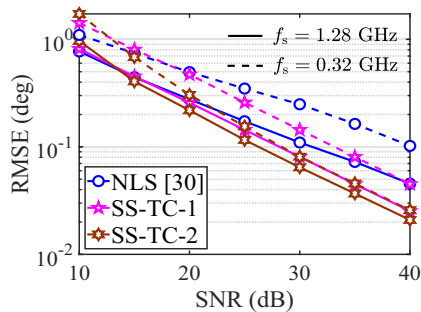

(c) Azimuth AoD

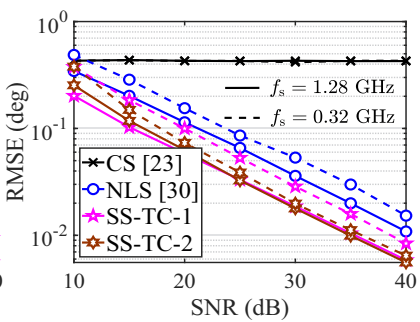

(b) Elevation AoA)

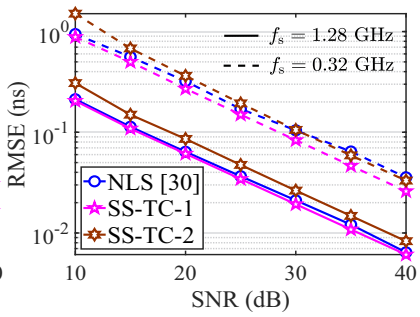

(d) Time Delay
Fig. 10. Parameter RMSE performance vs. SNR, $N_{\mathrm{x}}=N_{\mathrm{y}}=16$, MR $=82.5 \%, P=6, K=3$.

constant RMSE of AoA due to the fixed resolution of angle codebooks; the NLS method yields considerable parameter accuracy but worse channel NMSE, which indicates that it lacks sufficient stability to simultaneously obtain accurate estimation along the space/time/frequency domains.

Fig. 11 plots the NMSE curves versus the quantity of training subcarriers. It shows that as $K$ increases, the SSTC and NLS achieve evidently enhanced performance, while the CS and TMac improve only marginally. Moreover, one can observe that as the performance of SS-TC-1 continues to improve, the result of SS-TC-2 gradually converges to a threshold of $4 \times 10^{-4}$. This observation is consistent with the earlier frequency analysis of Fig. 9, 10, which suggests that there is no need to further increase the number of training subcarriers when the structural frequency information has been fully exploited. Furthermore, compared with Fig. 7, Fig. 11 


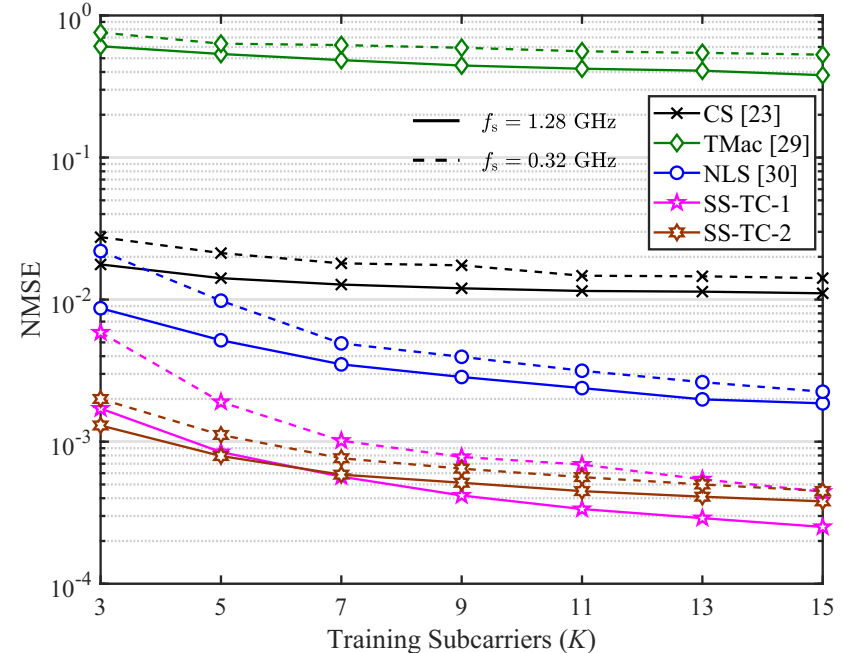

Fig. 11. Channel NMSE performance vs. training subcarriers, $N_{\mathrm{x}}=N_{\mathrm{y}}=$ $16, \mathrm{MR}=82.5 \%, P=6$, SNR $=30 \mathrm{~dB}$.

shows that the training subcarriers do not contribute as much as the training frames to the tensor signal completion, which can be explained that as $K, P$ increase to large values, the "regularly"-sampled $\mathbf{P}_{\tau}$ does not provide as much effective information as the randomly-sampled $\mathbf{G}_{\alpha}$.

Fig. 12 plots the ergodic spectral efficiency curves of the IRS-aided system versus the MR, which is defined as [6]

$$
\frac{1}{P K} \mathbb{E}\left\{\sum_{p, k} \log _{2}\left(1+\frac{P_{p, k}}{\sigma_{n}^{2}}\left\|\mathbf{H}_{p, k}^{\mathrm{BI}} \operatorname{Diag}\left(\mathbf{h}_{p, k}^{\mathrm{IM}}\right) \hat{\boldsymbol{\psi}}_{p, k}\right\|^{2}\right)\right\},
$$

where $P_{p, k}, \sigma_{n}^{2}$ denote the transmit power and noise variance, respectively; the IRS coefficients $\hat{\boldsymbol{\psi}}_{p, k}$ are generated by searching a codebook $\mathbf{D}_{\mathbf{x}} \otimes \mathbf{D}_{\mathbf{y}}$ with $\left[\mathbf{D}_{\mathbf{x}(\mathrm{y})}\right]_{m, n}=e^{-j(m-1) \frac{2 \pi}{N_{\mathbf{x}(y)}} n}$ based on the estimated $\widehat{\mathbf{H}}_{p, k}^{\mathrm{BM}} \triangleq \widehat{\mathbf{H}}_{p, k}^{\mathrm{BI}} \operatorname{Diag}\left(\hat{\mathbf{h}}_{p, k}^{\mathrm{IM}}\right)$ as in [23]. ${ }^{15}$ The figure illustrates that when the MR is less than $85.0 \%$, the SS-TC methods, especially the SS-TC-2 version, can achieve robust spectral efficiency close to that with perfect CSI. Moreover, the CS method yields considerable spectral efficiency thanks to its robust channel NMSE performance shown in Fig. 5, 6. As the MR gradually increases, the spectral efficiency performance of TMac and NLS dramatically deteriorates because the former cannot acquire effective channel estimates, and the latter suffers from estimation instability with a limited number of samples $N_{\mathrm{A}}$.

\section{Vi. Conclusions}

We considered the channel estimation of an IRS-assisted mmWave MIMO-OFDM system. We divided the cascaded channel estimation problem to the MS-to-IRS and BS-toIRS subproblems to avoid the inherent estimation ambiguities. We developed a hybrid IRS design composed of passive and active modules, balancing the requirements of effective

\footnotetext{
${ }^{15}$ In the simulation, the transmitted SNR $P_{t, k} / \sigma_{n}^{2}$ is approximated by the quotient of the received SNR $\rho$ and the maximal channel gain given the true codeword $\boldsymbol{\psi}_{p, k}^{\star}$ as $\rho /\left\|\mathbf{H}_{p, k}^{\mathrm{BM}} \boldsymbol{\psi}_{p, k}^{\star}\right\|^{2}$.
}

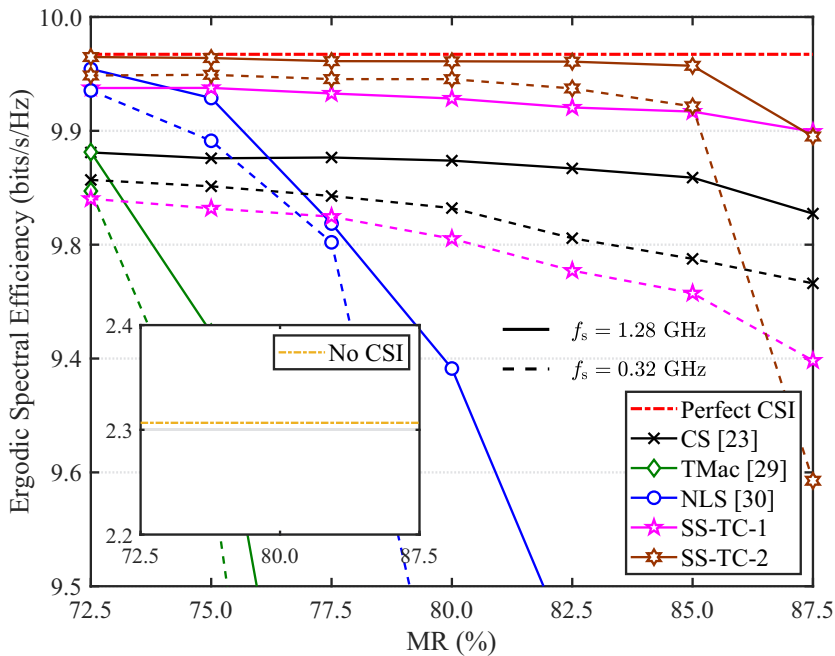

Fig. 12. Ergodic spectral efficiency performance vs. MR, $N_{\mathrm{x}}=N_{\mathrm{y}}=16$, $P=6, K=3, \mathrm{SNR}=30 \mathrm{~dB}$.

signal processing and hardware complexity. We modeled the narrowband training signal as an incomplete third-order CPD tensor and derived a fiber sampling-tensor completion problem. We developed an algebraic solution that applies to arbitrary valid design of hybrid layout. Then, we turned to the wideband OFDM training case, and established a fourth-order CPD tensor completion model. By leveraging the structural characteristic of training signals in the space and frequency domain, we proposed two different channel estimation and parameter recovery solutions. The uniqueness conditions of CPD tensor completion were also analyzed, which can inform the hybrid IRS design and training configuration. Numerical results showed that the proposed strategy outperforms the traditional schemes in terms of accuracy and complexity. In our future work, we are going to improve the algorithm performance against regular sampling designs.

\section{APPENDIX A}

\section{PRELIMINARIES OF TENSOR THEORY}

We present here some basic concepts of tensor algebra. Tensors are multi-dimensional data arrays. Vectors and matrices are one-dimensional and two-dimensional tensors, respectively.

Definition 1. $\mathcal{X} \triangleq\left[x_{i_{1}, \ldots, i_{N}}\right] \in \mathbb{C}^{I_{1} \times \cdots \times I_{N}}$ denotes a $N$ thorder tensor. The vector $\mathbf{x}_{i_{1}, \ldots, i_{n-1},:, i_{n+1}, \ldots, i_{N}} \in \mathbb{C}^{I_{n}}$ with all indices along the $n$th dimension is called a mode- $n$ fiber. The matrix $\mathbf{X}_{i_{1}, \ldots, i_{n_{1}-1,:}, i_{n_{1}+1}, \ldots, i_{n_{2}-1},:, i_{n_{2}+1}, \ldots, i_{N}} \in \mathbb{C}^{I_{n_{1}} \times I_{n_{2}}}$ with all indices along the $n_{1}, n_{2}$ th dimensions is called a mode- $\left(n_{1}, n_{2}\right)$ slice.

Definition 2 [25]-[27]. A Canonical Polyadic Decomposition $(C P D)$ is a factorization of $\mathcal{X}$ into a sum of rank-1 terms:

$$
\begin{aligned}
\mathcal{X} & =\sum_{r=1}^{R} \mathbf{a}_{r}^{(1)} \circ \mathbf{a}_{r}^{(2)} \circ \cdots \circ \mathbf{a}_{r}^{(N)} \\
& =\llbracket \mathbf{A}^{(1)}, \mathbf{A}^{(2)}, \ldots, \mathbf{A}^{(N)} \rrbracket,
\end{aligned}
$$

where $R$ is the rank of $\mathcal{X}$, i.e., the minimal number of rank1 tensors that yield $\mathcal{X}$ in a linear combination. The matrices 
$\mathbf{A}^{(n)}=\left[\mathbf{a}_{1}^{(n)}, \ldots, \mathbf{a}_{R}^{(n)}\right] \in \mathbb{C}^{I_{n} \times R}, \forall n$ are referred to as the factor matrices of CPD in (38).

Definition 3 [47]. The matricization operation denoted by $\operatorname{Matr}\left(\mathcal{X} ;\left[k_{1}, \ldots, k_{P}\right],\left[k_{P+1}, \ldots, k_{N}\right]\right)$ unfolds $\mathcal{X}$ into a ma$\operatorname{trix} \mathbf{X} \in \mathbb{C}^{P} P=1 I_{k_{p}} \times \prod_{q=P+1}^{N} I_{k_{q}}$ as:

$$
\begin{aligned}
& \widetilde{\mathcal{X}}=\operatorname{permute}\left(\boldsymbol{\mathcal { X }} ;\left[k_{1}, \ldots, k_{N}\right]\right), \\
& \mathbf{X}=\operatorname{reshape}\left(\widetilde{\mathcal{X}} ;\left[\prod_{p=1}^{P} I_{k_{p}}, \prod_{q=P+1}^{N} I_{k_{q}}\right]\right),
\end{aligned}
$$

where $\tilde{\mathcal{X}} \in \mathbb{C}^{I_{k_{1}} \times \cdots \times I_{k_{N}}} ; \operatorname{permute}\left(\mathcal{X} ; \mathbf{t}_{\text {ord }}^{T}\right)$ rearranges the dimensions of tensor $\mathcal{X}$ in the order specified by $\mathbf{t}_{\text {ord }}$; reshape $\left(\mathcal{X} ; \mathbf{t}_{\text {size }}^{T}\right)$ reconstructs $\mathcal{X}$ into an array $\mathbf{X}$ with sizes specified by $\mathbf{t}_{\text {size }}$.

As an inverse transform, the tensorization operation denoted by $\operatorname{Tens}\left(\mathbf{X} ;\left[I_{1}, \ldots, I_{N}\right],\left[k_{1}, \ldots, k_{P}\right],\left[k_{P+1}, \ldots, k_{N}\right]\right)$ tensorizes $\mathbf{X}$ to a tensor $\mathcal{X}$ as:

$$
\begin{aligned}
\widetilde{\mathcal{X}} & =\operatorname{reshape}\left(\mathbf{X} ;\left[I_{k_{1}}, \ldots, I_{k_{N}}\right]\right), \\
\mathcal{X} & =\operatorname{permute}\left(\widetilde{\mathcal{X}} ; \mathbf{t}_{\text {reord }}^{T}\right),
\end{aligned}
$$

where $\left[\mathbf{t}_{\text {reord }}\right]_{k_{n}}=n, \forall n$ specifies the reordering indices. The above functions share the identical definitions of their counterparts in MATLAB. ${ }^{16}$

Lemma 1 [44]. Consider a CPD tensor $\mathcal{X} \in \mathbb{C}^{I_{1} \times \cdots \times I_{N}}$ with factor matrices $\mathbf{A}^{(n)} \in \mathbb{C}^{I_{n} \times R}, \forall n \in \mathcal{I}(N)$. One can verify the relationship between the matricization result of CPD model and its factor matrices as

$$
\begin{aligned}
\mathbf{X} & =\operatorname{Matr}\left(\boldsymbol{\mathcal { X }} ;\left[k_{1}, \ldots, k_{P}\right],\left[k_{P+1}, \ldots, k_{N}\right]\right) \\
& =\left(\mathbf{A}^{\left(k_{P}\right)} \odot \cdots \odot \mathbf{A}^{\left(k_{1}\right)}\right)\left(\mathbf{A}^{\left(k_{N}\right)} \odot \cdots \odot \mathbf{A}^{\left(k_{P+1}\right)}\right)^{T} .
\end{aligned}
$$

\section{APPENDIX B \\ ProOF OF THEOREM 2}

We perform the SVD of (29) as $\mathbf{Z}_{\text {sub }}=\mathbf{U}_{\text {sub }} \boldsymbol{\Sigma}_{\text {sub }} \mathbf{V}_{\text {sub }}^{H}$, where $\mathbf{U}_{\text {sub }} \in \mathbb{C}^{N_{\mathrm{A}} \times L}, \boldsymbol{\Sigma}_{\text {sub }} \in \mathbb{C}^{L \times L}$ and $\mathbf{V}_{\text {sub }} \in \mathbb{C}^{K P \times L}$. If $\boldsymbol{\Xi}\left(\mathbf{A}_{\mathrm{x}}, \mathbf{A}_{\mathrm{y}}\right)$ and $\mathbf{P}_{\tau}^{\left(K^{\prime}-1\right)} \odot \mathbf{G}_{\beta}$ have full column rank, there exists a nonsingular matrix $\mathbf{F} \in \mathbb{C}^{L \times L}$ such that

$$
\mathbf{U}_{\mathrm{sub}} \boldsymbol{\Sigma}_{\mathrm{sub}} \mathbf{F}^{-T}=\mathbf{\Xi}\left(\mathbf{A}_{\mathrm{x}}, \mathbf{A}_{\mathrm{y}}\right), \mathbf{V}_{\mathrm{sub}}^{*} \mathbf{F}=\mathbf{P}_{\tau} \odot \mathbf{G}_{\beta} .
$$

By leveraging the rotational-invariance characteristic of $\mathbf{P}_{\tau}$, we have

$$
\begin{aligned}
\mathbf{V}_{\mathrm{sub}, 1}^{*} \mathbf{F} & =\mathbf{P}_{\tau}^{(K-1)} \odot \mathbf{G}_{\beta}, \\
\mathbf{V}_{\mathrm{sub}, 2}^{*} \mathbf{F} & =\left(\mathbf{P}_{\tau}^{(K-1)} \odot \mathbf{G}_{\beta}\right) \boldsymbol{\Delta}_{\tau},
\end{aligned}
$$

where $\mathbf{V}_{\text {sub }, 1} \triangleq\left[\mathbf{V}_{\text {sub }}\right]_{1:(K-1) P,:}, \mathbf{V}_{\text {sub }, 2} \triangleq\left[\mathbf{V}_{\text {sub }}\right]_{P+1: K P,:} ;$ $\boldsymbol{\Delta}_{\tau} \triangleq \operatorname{Diag}\left(\left[e^{j \omega_{\tau, 1}}, \ldots, e^{j \omega_{\tau, L}}\right]^{T}\right)$ with $\omega_{\tau, \ell} \triangleq-2 \pi f_{\mathrm{s}} \tau_{\ell} / K_{\mathrm{s}}$. $\boldsymbol{\Delta}_{\tau}$ and $\mathbf{F}$ can be derived by the GEVD method as

$$
\mathbf{F} \boldsymbol{\Delta}_{\tau} \mathbf{F}^{-1}=\left(\mathbf{V}_{\text {sub }, 1}^{*}\right)^{\dagger} \mathbf{V}_{\text {sub }, 2}^{*} .
$$

If $\boldsymbol{\Delta}_{\tau}$ contains distinct diagonal entries, $\mathbf{G}_{\beta}$ can be columnwisely computed as [48]

$$
\mathbf{g}_{\beta, \ell}=\left(\frac{\mathbf{p}_{\tau, \ell}^{H}}{\left\|\mathbf{p}_{\tau, \ell}\right\|_{2}^{2}} \otimes \mathbf{I}_{P}\right) \mathbf{V}_{\mathrm{sub}}^{*} \mathbf{f}_{\ell} .
$$

\footnotetext{
${ }^{16} \mathrm{http}: / /$ www.mathworks.com/help/matlab/ref/reshape(permute).html.
}

One can compute $\boldsymbol{\Xi}\left(\mathbf{A}_{\mathrm{x}}, \mathbf{A}_{\mathrm{y}}\right)$ by (42), and recover $\left\{\mathbf{a}_{\mathrm{x}, \ell}, \mathbf{a}_{\mathrm{y}, \ell}\right\}$ following (21)-(24).

\section{APPENDIX C \\ PRoOF OF THEOREM 3}

We perform a matricization of $\mathcal{Z}$ in (29) as

$$
\begin{aligned}
\widetilde{\mathbf{Z}} & \triangleq \operatorname{Matr}(\mathcal{W} ;[1,3],[4,2]) * \operatorname{Matr}(\mathcal{Y} ;[1,3],[4,2]) \\
& =\widetilde{\mathbf{W}} *\left(\left(\mathbf{P}_{\tau} \odot \mathbf{A}_{\mathbf{x}}\right)\left(\mathbf{A}_{\mathbf{y}} \odot \mathbf{G}_{\beta}\right)^{T}\right)
\end{aligned}
$$

where $\widetilde{\mathbf{W}} \in\{0,1\}^{K N_{\mathrm{x}} \times P N_{\mathrm{y}}}$ is the matricization result of $\mathcal{W}$. Then, we perform a spatial smoothing transformation of (46) with $K_{\mathrm{x}}+K_{\mathrm{y}}=K+1$ as

$$
\begin{aligned}
& \widetilde{\mathbf{Z}}_{\mathrm{ss}} \triangleq\left[\begin{array}{llll}
\mathbf{J}_{1} \widetilde{\mathbf{Z}} & \mathbf{J}_{2} \widetilde{\mathbf{Z}} & \ldots & \mathbf{J}_{K_{\mathrm{y}}} \widetilde{\mathbf{Z}}
\end{array}\right], \\
& \mathbf{J}_{k_{\mathrm{y}}} \triangleq\left[\begin{array}{lll}
\mathbf{0}_{K_{\mathrm{x}} \times\left(k_{\mathrm{y}}-1\right)} & \mathbf{I}_{K_{\mathrm{x}}} & \mathbf{0}_{K_{\mathrm{x}} \times\left(K_{\mathrm{y}}-k_{\mathrm{y}}\right)}
\end{array}\right] \otimes \mathbf{I}_{N_{\mathrm{x}}} .
\end{aligned}
$$

By leveraging the rotational-invariance feature of $\mathbf{P}_{\tau}$, we have [45], [49]

$$
\begin{aligned}
\widetilde{\mathbf{Z}}_{\mathrm{ss}} & =\widetilde{\mathbf{W}}_{\mathrm{ss}} *\left(\left(\mathbf{P}_{\tau}^{\left(K_{\mathrm{x}}\right)} \odot \mathbf{A}_{\mathrm{x}}\right)\left(\mathbf{P}_{\tau}^{\left(K_{\mathrm{y}}\right)} \odot \mathbf{A}_{\mathrm{y}} \odot \mathbf{G}_{\beta}\right)^{T}\right) \\
& =\widetilde{\mathbf{W}}_{\mathrm{ss}} *\left(\widetilde{\mathbf{A}}_{\mathrm{x}}\left(\widetilde{\mathbf{A}}_{\mathrm{y}} \odot \mathbf{G}_{\beta}\right)^{T}\right),
\end{aligned}
$$

where $\widetilde{\mathbf{W}}_{\mathrm{sS}} \in\{0,1\}^{K_{\mathrm{x}} N_{\mathrm{x}} \times K_{\mathrm{y}} N_{\mathrm{y}} P}$ is the spatial smoothing result of $\widehat{\mathbf{W}}$. The equivalent incomplete signal tensor $\widetilde{\mathcal{Z}}_{\mathrm{ss}} \in$ $\mathbb{C}^{K_{\mathrm{x}} N_{\mathrm{x}} \times K_{\mathrm{y}} N_{\mathrm{y}} \times P}$ can be derived from (48) as

$$
\begin{aligned}
\widetilde{\mathcal{Z}}_{\mathrm{ss}} & \triangleq \operatorname{Tens}\left(\widetilde{\mathbf{Z}}_{\mathrm{ss}} ;\left[K_{\mathrm{x}} N_{\mathrm{x}}, K_{\mathrm{y}} N_{\mathrm{y}}, P\right], 1,[3,2]\right) \\
& =\widetilde{\mathcal{W}}_{\mathrm{ss}} * \llbracket \widetilde{\mathbf{A}}_{\mathrm{x}}, \widetilde{\mathbf{A}}_{\mathrm{y}}, \mathbf{G}_{\beta} \rrbracket,
\end{aligned}
$$

where $\widetilde{\mathcal{W}}_{\mathrm{ss}} \in\{0,1\}^{K_{\mathrm{x}} N_{\mathrm{x}} \times K_{\mathrm{y}} N_{\mathrm{y}} \times P}$ is the tensorization result of $\widetilde{\mathbf{W}}_{\mathrm{ss}}$. This CPD tensor completion problem can be solved up to scaling and permutation ambiguities via (14)-(24). One can separate $\left\{\mathbf{A}_{\mathbf{x}}, \mathbf{A}_{\mathrm{y}}, \mathbf{P}_{\tau}\right\}$ from $\left\{\widetilde{\mathbf{A}}_{\mathbf{x}}, \widetilde{\mathbf{A}}_{\mathrm{y}}\right\}$ by leveraging their rotational-invariance feature as

$$
\begin{aligned}
e^{j \omega_{\mathrm{x}, \ell}} & =\left[\boldsymbol{\Pi}_{\mathbf{x}} \tilde{\mathbf{a}}_{\mathrm{x}, \ell}\right]_{1:\left(N_{\mathrm{x}}-1\right) K_{\mathrm{x}}}^{\dagger}\left[\boldsymbol{\Pi}_{\mathrm{x}} \tilde{\mathbf{a}}_{\mathrm{x}, \ell}\right]_{K_{\mathrm{x}}+1: N_{\mathrm{x}} K_{\mathrm{x}}}, \\
e^{j \omega_{\mathrm{y}, \ell}} & =\left[\boldsymbol{\Pi}_{\mathrm{y}} \tilde{\mathbf{a}}_{\mathrm{y}, \ell}\right]_{1:\left(N_{\mathrm{y}}-1\right) K_{\mathrm{y}}}^{\dagger}\left[\boldsymbol{\Pi}_{\mathrm{y}} \tilde{\mathbf{a}}_{\mathrm{y}, \ell}\right]_{K_{\mathrm{y}}+1: N_{\mathrm{y}} K_{\mathrm{y}}}, \\
e^{j \omega_{\tau, \ell}} & =\left[\tilde{\mathbf{a}}_{\mathbf{x}, \ell}\right]_{1:\left(K_{\mathrm{x}}-1\right) N_{\mathrm{x}}}^{\dagger}\left[\tilde{\mathbf{a}}_{\mathrm{x}, \ell}\right]_{N_{\mathrm{x}}+1: K_{\mathrm{x}} N_{\mathrm{x}}} \\
& =\left[\tilde{\mathbf{a}}_{\mathrm{y}, \ell}\right]_{1:\left(K_{\mathrm{y}}-1\right) N_{\mathrm{y}}}^{\dagger}\left[\tilde{\mathbf{a}}_{\mathrm{y}, \ell}\right]_{N_{\mathrm{y}}+1: K_{\mathrm{y}} N_{\mathrm{y}}},
\end{aligned}
$$

where the phases $\omega_{x, \ell} \triangleq \sin \phi_{\ell} \cos \theta_{\ell}, \omega_{y, \ell} \triangleq \sin \phi_{\ell} \sin \theta_{\ell}$ and $\omega_{\tau, \ell} \triangleq-2 \pi f_{\mathrm{s}} \tau_{\ell} / K_{\mathrm{s}}$ are called the generators of $\mathbf{A}_{\mathrm{x}}$, $\mathbf{A}_{\mathrm{y}}$ and $\mathbf{P}_{\tau}$, respectively. $\Pi_{\mathrm{x}} \in\{0,1\}^{K_{\mathrm{x}} N_{\mathrm{x}} \times K_{\mathrm{x}} N_{\mathrm{x}}}$ and $\Pi_{\mathrm{y}} \in\{0,1\}^{K_{\mathrm{y}} N_{\mathrm{y}} \times K_{\mathrm{y}} N_{\mathrm{y}}}$ are permutation matrices such that $\boldsymbol{\Pi}_{\mathrm{x}} \widetilde{\mathbf{A}}_{\mathbf{x}}=\mathbf{A}_{\mathbf{x}} \odot \mathbf{P}_{\tau}^{\left(K_{\mathrm{x}}\right)}$ and $\boldsymbol{\Pi}_{\mathrm{y}} \widetilde{\mathbf{A}}_{\mathrm{y}}=\mathbf{A}_{\mathrm{y}} \odot \mathbf{P}_{\tau}^{\left(K_{\mathrm{y}}\right)}$, respectively.

\section{REFERENCES}

[1] T. S. Rappaport et al., "Millimeter wave mobile communications for 5G cellular: it will work!," IEEE Access, vol. 1, pp. 335-349, May 2013.

[2] M. Matthaiou, O. Yurduseven, H. Q. Ngo, D. Morales-Jimenez, S. L. Cotton, and V. F. Fusco, "The road to 6G: Ten physical layer challenges for communications engineers," IEEE Commun. Mag., Early Access, 2021.

[3] S. Nie, J. M. Jornet, and I. F. Akyildiz, "Intelligent environments based on ultra-massive MIMO platforms for wireless communication in millimeter wave and terahertz bands," in Proc. IEEE ICASSP, Apr. 2019, pp. 7849-7853. 
[4] S. Hu, F. Rusek, and O. Edfors, "Beyond massive MIMO: The potential of data transmission with large intelligent surfaces," IEEE Trans. Signal Process., vol. 66, no. 10, pp. 2746-2758, May 2018.

[5] N. Rajatheva et al., "White paper on broadband connectivity in 6G," arXiv preprint, arXiv: 2004.14247, Apr. 2020.

[6] Y. Han, W. Tang, S. Jin, C. Wen, and X. Ma, "Large intelligent surfaceassisted wireless communication exploiting statistical CSI," IEEE Trans. Veh. Technol., vol. 68, no. 8, pp. 8238-8242, Aug. 2019.

[7] Q. Wu and R. Zhang, "Towards smart and reconfigurable environment: Intelligent reflecting surface aided wireless network," IEEE Commun. Mag., vol. 58, no. 1, pp. 106-112, Jan. 2020.

[8] W. Tang et al., "Wireless communications with programmable metasurface: Transceiver design and experimental results," China Commun., vol. 16, no. 5, pp. 46-61, Jun. 2019.

[9] Q. Wu and R. Zhang, "Intelligent reflecting surface enhanced wireless network: joint active and passive beamforming design," in Proc. IEEE GLOBECOM, Dec. 2018, pp. 1-6.

[10] J. Chen, Y.-C. Liang, Y. Pei, and H. Guo, "Intelligent reflecting surface: A programmable wireless environment for physical layer security," IEEE Access, vol. 7, pp. 82599-82612, Jun. 2019.

[11] Q. Wu and R. Zhang, "Weighted sum power maximization for intelligent reflecting surface aided SWIPT," IEEE Wireless Commun. Lett., vol. 9, no. 5, pp. 586-590, May 2020 .

[12] L. Zhang, Y. Wang, W. Tao, Z. Jia, T. Song, and C. Pan, "Intelligent reflecting surface aided MIMO cognitive radio systems," IEEE Trans. Veh. Tech., vol. 69, no. 10, pp. 11445-11457, Oct. 2020.

[13] T. Hou, Y. Liu, Z. Song, X. Sun, Y. Chen, and L. Hanzo, "Reconfigurable intelligent surface aided NOMA networks," IEEE J. Sel. Areas Commun., vol. 38, no. 11, pp. 2575-2588, Nov. 2020.

[14] D. Mishra and H. Johansson, "Channel estimation and low-complexity beamforming design for passive intelligent surface assisted MISO wireless energy transfer," in Proc. IEEE ICASSP, Apr. 2019, pp. 4659-4663.

[15] Z. He and X. Yuan, "Cascaded channel estimation for large intelligent metasurface assisted massive MIMO," IEEE Wireless Commun. Lett., vol. 9, no. 2, pp. 210-214, Feb. 2020.

[16] J. He, H. Wymeersch, and M. Juntti, "Channel estimation for RISaided mmWave MIMO systems via atomic norm minimization," arXiv preprint, arXiv: 2007.08158, Jul. 2020.

[17] J. Chen, Y. Liang, H. V. Cheng, and W. Yu, "Channel estimation for reconfigurable intelligent surface aided multi-user MIMO systems," arXiv preprint, arXiv: 1912.03619, Dec. 2019.

[18] B. Ning, Z. Chen, W. Chen, and Y. Du, "Channel estimation and transmission for intelligent reflecting surface assisted THz communications," in Proc. IEEE ICC, Jun. 2020, pp. 1-7.

[19] A. M. Elbir, A. Papazafeiropoulos, P. Kourtessis, and S. Chatzinotas, "Deep channel learning for large intelligent surfaces aided mm-wave massive MIMO systems,", IEEE Wireless Commun. Lett., vol. 9, no. 9, pp. 1447-1451, Sept. 2020.

[20] G. T. de Araújo and A. L. F. de Almeida, "PARAFAC-based channel estimation for intelligent surface assisted MIMO system," in Proc. IEEE SAM, Jun. 2020, pp. 1-5.

[21] L. Wei, C. Huang, G. C. Alexandropoulos, and C. Yuen, "Parallel factor decomposition channel estimation in RIS-assisted multi-user MISO communication,", in Proc. IEEE SAM. Jun. 2020, pp. 1-5.

[22] X. Guan, Q. Wu, and R. Zhang, "Anchor-assisted intelligent reflecting surface channel estimation for multiuser communications," arXiv preprint, arXiv: 2008.00622, Aug. 2020.

[23] A. Taha, M. Alrabeiah, and A. Alkhateeb, "Enabling large intelligent surfaces with compressive sensing and deep learning," arXiv preprint, arXiv: 1904.10136, Apr. 2019.

[24] S. Zhang, S. Zhang, F. Gao, J. Ma, and O. A. Dobre, "Deep learning optimized sparse antennas activation for reconfigurable intelligent surface assisted communication," arXiv preprint, arXiv: 2009.01607, Sept. 2020.

[25] I. Domanov and L. De Lathauwer, "On the uniqueness of the canonical polyadic decomposition of third-order tensors-Part I: Basic results and uniqueness of one factor matrix," SIAM J. Matrix Anal. Appl., vol. 34, no. 3, pp. 855-875, Jul. 2013.

[26] I. Domanov and L. De Lathauwer, "On the uniqueness of the canonical polyadic decomposition of third-order tensors-Part II: Uniqueness of the overall decomposition," SIAM J. Matrix Anal. Appl., vol. 34, no. 3, pp. 876-903, Jul. 2013.

[27] I. Domanov and L. De Lathauwer, "Canonical polyadic decomposition of third-order tensors: Relaxed uniqueness conditions and algebraic algorithm," Linear Algebra Appl., vol. 513, pp. 342-375, Jan. 2017.
[28] L. Karlsson, D. Kressner, and A. Uschmajew, "Parallel algorithms for tensor completion in the CP format," Parallel Comput., vol. 57, pp. 222-234, Sept. 2016

[29] Y. Xu, R. Hao, W. Yin, and Z. Su, "Parallel matrix factorization for low-rank tensor completion," Inverse Probl. Imag., vol. 9, no. 2, pp. 601-624, Dec. 2015.

[30] L. Sorber, M. Van Barel, and L. De Lathauwer, "Structured data fusion," IEEE J. Sel. Topics Signal Process., vol. 9, no. 4, pp. 586-600, Jun. 2015.

[31] S. Abeywickrama, R. Zhang, Q. Wu, and C. Yuen, "Intelligent reflecting surface: Practical phase shift model and beamforming optimization," IEEE Trans. Commun., vol. 68, no. 9, pp. 5849-5863, Sept. 2020.

[32] W. Cai, H. Li, M. Li, and Q. Liu, "Practical modelling and beamforming for intelligent reflecting surface aided wideband systems," IEEE Commun. Lett., vol. 24, no. 7, pp. 1568-1571, Jul. 2020.

[33] W. Tang, et al., "MIMO transmission through reconfigurable intelligent surface: System design, analysis, and implementation," IEEE J. Sel. Areas Commun., vol. 38, no. 11, pp. 2683-2699, Nov. 2020.

[34] H. Wymeersch, J. He, B. Denis, A. Clemente, and M. Juntti, "Radio localization and mapping with reconfigurable intelligent surfaces," arXiv preprint, arXiv: 1912.09401, Jun. 2020.

[35] S. Hu, F. Rusek, and O. Edfors, "Beyond massive MIMO: The potential of positioning with large intelligent surfaces," IEEE Trans. Signal Process., vol. 66, no. 7, pp. 1761-1774, Jan. 2018.

[36] S. E. Zegrar, L. Afeef, and H. Arslan, "A general framework for RIS-aided mmWave communication networks: Channel estimation and mobile user tracking," arXiv preprint, arXiv: 2009.01180, Sept. 2020.

[37] E. Björnson, Ö. Özdogan, and E. G. Larsson, "Intelligent reflective surface versus decode-and-forward: How large surfaces are needed to beat relaying?" IEEE Wireless Commun. Lett., vol. 9, no. 2, pp. 244248, Feb. 2020.

[38] C. Huang, A. Zappone, G. C. Alexandropoulos, M. Debbah, and C. Yuen, "Reconfigurable intelligent surfaces for energy efficiency in wireless communication," IEEE Trans. Wireless Commun., vol. 18, no. 8, pp. 4157-4170, Aug. 2019.

[39] R. W. Heath, Jr., N. González-Prelcic, S. Rangan, W. Roh, and A. M. Sayeed, "An overview of signal processing techniques for millimeter wave MIMO systems," IEEE J. Sel. Topics Signal Process., vol. 10, no. 3, pp. 436-453, Apr. 2016.

[40] Q. Qin, L. Gui, P. Cheng, and B. Gong, "Time-varying channnel estimation for millimeter wave multiuser MIMO systems," IEEE Trans. Veh. Tech., vol. 67, no. 10, pp. 9435-9448, Oct. 2018

[41] M. Sørensen and L. De Lathauwer, "Fiber sampling approach to canonical polyadic decomposition and application to tensor completion," SIAM J. Matrix Anal. Appl., vol. 40, no. 3, pp. 888-917, Jul. 2019.

[42] C. I. Kanatsoulis, X. Fu, N. D. Sidiropoulos, and M. Akçakaya, "Tensor completion from regular sub-Nyquist samples," IEEE Trans. Signal Process., vol. 68, pp. 1-16, Nov. 2019.

[43] K. Liu, J. P. C. L. da Costa, H. C. So, L. Huang, and J. Ye, "Detection of number of components in CANDECOMP/ PARAFAC models via minimum description length," Digital Signal Process., vol. 51, pp. 110123, Jan. 2016

[44] I. Domanov and L. De Lathauwer, "Canonical polyadic decomposition of third-order tensors: Reduction to generalized eigenvalue decomposition," SIAM J. Matrix Anal. Appl., vol. 35, no. 2, pp. 636-660, May 2014.

[45] Y. Lin, S. Jin, M. Matthaiou, and X. You, "Tensor-based channel estimation for millimeter wave MIMO-OFDM with dual-wideband effects," IEEE Trans. Commun., vol. 68, no. 7, pp. 4218-4232, Mar. 2020.

[46] A. J. Van Der Veen and A. Paulraj, "An analytical constant modulus algorithm," IEEE Trans. Signal Process., vol. 44, no. 5, pp. 1136-1155, May 1996.

[47] N. Vervliet, O. Debals, L. Sorber, M. Van Barel, and L. De Lathauwer, Tensorlab 3.0, [Available Online], Mar. 2016. URL: https://www.tensorlab.net/.

[48] M. Sørensen and L. De Lathauwer, "Blind signal separation via tensor decomposition with Vandermonde factor: Canonical polyadic decomposition," IEEE Trans. Signal Process., vol. 61, no. 22, pp. 5507-5519, Nov. 2013.

[49] J. Liu, X. Liu, and X. Ma, "Multidimensional frequency estimation with finite snapshots in the presence of identical frequencies," IEEE Trans. Signal Process., vol. 55, no. 11, pp. 5179-5194, Nov. 2007. 


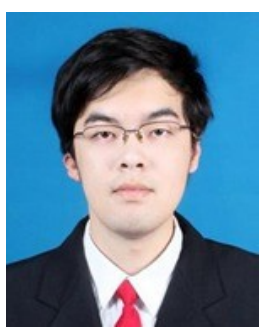

Yuxing Lin (S'18) received the B.S. degree in information engineering from Southeast University, Nanjing, China, in 2016. He is currently pursuing the Ph.D. degree in the School of Information Science and Engineering at Southeast University. His research interests include massive MIMO, millimeter wave wireless communications, hybrid beamforming, channel estimation, beam training/tracking, tensor signal processing and intelligent reconfigurable surface-assisted communications.

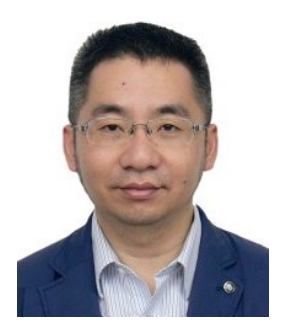

Shi Jin (S'06-M'07-SM'17) received the B.S. degree in communications engineering from Guilin University of Electronic Technology, Guilin, China, in 1996, the M.S. degree from Nanjing University of Posts and Telecommunications, Nanjing, China, in 2003, and the Ph.D. degree in information and communications engineering from the Southeast University, Nanjing, in 2007. From June 2007 to October 2009, he was a Research Fellow with the Adastral Park Research Campus, University College London, London, U.K. He is currently with the faculty of the National Mobile Communications Research Laboratory, Southeast University. His research interests include space time wireless communications, random matrix theory, and information theory. He serves as an Associate Editor for the IEEE TRANSACTIONS ON WiRELESS COMMUNICATIONS, and IEEE COMMUNiCATIONS LeTtERs, and IET COMMUniCATIONS. Dr. Jin and his co-authors have been awarded the 2011 IEEE Communications Society Stephen O. Rice Prize Paper Award in the field of communication theory and a 2010 Young Author Best Paper Award by the IEEE Signal Processing Society.

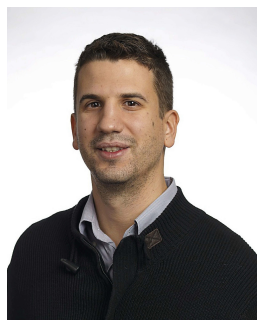

Michail Matthaiou (S'05-M'08-SM'13) was born in Thessaloniki, Greece in 1981. He obtained the Diploma degree (5 years) in Electrical and Computer Engineering from the Aristotle University of Thessaloniki, Greece in 2004. He then received the M.Sc. (with distinction) in Communication Systems and Signal Processing from the University of Bristol, U.K. and Ph.D. degrees from the University of Edinburgh, U.K. in 2005 and 2008, respectively. From September 2008 through May 2010, he was with the Institute for Circuit Theory and Signal Processing, Munich University of Technology (TUM), Germany working as a Postdoctoral Research Associate. He is currently a Professor of Communications Engineering and Signal Processing and Deputy Director of the Centre for Wireless Innovation (CWI) at Queen's University Belfast, U.K. after holding an Assistant Professor position at Chalmers University of Technology, Sweden. His research interests span signal processing for wireless communications, massive MIMO systems, hardware-constrained communications, mmwave/THz systems and deep learning for communications.

Dr. Matthaiou and his coauthors received the IEEE Communications Society (ComSoc) Leonard G. Abraham Prize in 2017. He currently holds the ERC Consolidator Grant BEATRICE (2021-2026) focused on the interface between information and electromagnetic theories. He was awarded the prestigious 2018/2019 Royal Academy of Engineering/The Leverhulme Trust Senior Research Fellowship and also received the 2019 EURASIP Early Career Award. His team was also the Grand Winner of the 2019 Mobile World Congress Challenge. He was the recipient of the 2011 IEEE ComSoc Best Young Researcher Award for the Europe, Middle East and Africa Region and a co-recipient of the 2006 IEEE Communications Chapter Project Prize for the best M.Sc. dissertation in the area of communications. He has co-authored papers that received best paper awards at the 2018 IEEE WCSP and 2014 IEEE ICC and was an Exemplary Reviewer for IEEE COMMUNICATIONS LETTERS for 2010. In 2014, he received the Research Fund for International Young Scientists from the National Natural Science Foundation of China. $\mathrm{He}$ is currently the Editor-in-Chief of Elsevier Physical Communication, a Senior Editor for IEEE WIRELESS COMMUNICATIONS LETTERS and an Associate Editor for the IEEE JSAC SERIES ON MACHINE LEARNING FOR COMMUNiCATIONS AND NETWORKs. In the past, he was an Associate Editor for the IEEE TRANSACTIONS ON COMMUNICATIONS and Associate Editor/Senior Editor for IEEE COMMUNICATIONS LETTERS.

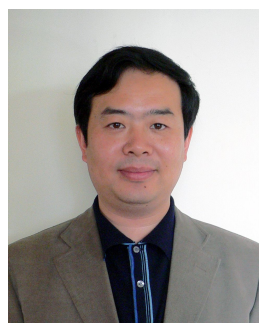

Xiaohu You (F'12) received the B.S., M.S., and $\mathrm{Ph} . \mathrm{D}$. degrees in electrical engineering from the Nanjing Institute of Technology, Nanjing, China, in 1982, 1985, and 1989, respectively. From 1987 to 1989, he was a Lecturer with the Nanjing Institute of Technology. Since 1990, he has been with Southeast University, first as an Associate Professor and then as a Professor. His research interests include mobile communications, adaptive signal processing, and artificial neural networks with applications to communications and biomedical engineering. $\mathrm{He}$ contributed over 40 IEEE journal papers and two books in the areas of adaptive signal processing and neural networks and their applications to communication systems. He was the Premier Foundation Investigator of the China National Science Foundation. From 1999 to 2002, he was the Principal Expert of the C3G Project, responsible for organizing China's 3G mobile communications research and development activities. From 2001 to 2006, he was the Principal Expert of the National 863 FuTURE Project. He received the Excellent Paper Award from the China Institute of Communications in 1987 and the Elite Outstanding Young Teacher Award from Southeast University in 1990, 1991, and 1993. He is currently the Chairman of the IEEE Nanjing Section. In 2012, he was selected as an IEEE Fellow for his contributions to the development of mobile communications in China. 